\title{
Models for passively mode-locked fiber lasers
}

\author{
François Sanchez, Hervé Leblond, Mohamed Salhi, Andrey Komarov ${ }^{1}$, Adil Haboucha \\ Laboratoire POMA, Université d'Angers, 2 Bd Lavoisier, 49000 Angers, France
}

e-mail : francois.sanchez@ univ-angers.fr, Tel : +332 41735447

\begin{abstract}
We give a review of theoretical approaches we have recently developed for the description of passively mode-locked fiber lasers. Mode-locking is assumed to be obtained with nonlinear polarization rotation. The first approach takes the form of a cubic Ginzburg-Landau equation where the coefficients take into account the orientation of the phase plates. A fully analytical study can be performed. The second model includes gain saturation allowing to investigate multiple pulse behaviour and multistability phenomena. Under some approximations this model reduces to a complex quintic Ginzburg-Landau equation. Passive harmonic modelocking can also be obtained with a suitable adaptation of the model.
\end{abstract}

13 November 2007

\footnotetext{
${ }^{1}$ Permanent address : Institute of Automation and Electrometry, Russian Academy of Sciences, Acad. Koptyug Pr., 1, 630090 Novosibirsk, Russia.
} 


\section{I - Introduction}

Passively mode-locked fiber lasers are very attractive from the dynamical point of view because they exhibit a large variety of behaviours. Indeed, in addition to the regular modelocking regime which has been reported in different optical configurations, many regimes involving several pulses by cavity round-trip have been observed or theoretically predicted. First experimental results were obtained with erbium-doped figure eight lasers [1-6]. It was observed bunches of pulses, pulses randomly spaced but well separated and also harmonic mode-locking, where the pulses are equally separated along the cavity. Other features such as Q-switch operation and bistability between the continuous and mode-lock regimes were also reported $[4,5]$. The evolution of the number of pulses as a function of the pump power was investigated in [5] where the authors showed that the pulses disappear one by one when the pump was decreased. Mode-locking using the figure eight geometry has been also reported with praseodymium [7] and ytterbium [8] doped fibers. Bunches of pulses were also observed [7]. The possibility of exploiting the nonlinear polarization rotation to obtain passive modelocking was first proposed by L. Dahlström [9] and a first theoretical description was given in [10]. Experimental demonstration of mode-locking through nonlinear polarization rotation was then demonstrated [11-13]. Here again, multiple pulsing was also observed although the basic principle of mode-locking was different from the one of figure eight lasers. In order to increase the energy per pulse the stretched-pulse configuration was proposed, it also allows to reduce the pulse duration [14]. This configuration leads to various dynamical behaviours such as bistability between the continuous (CW) and the mode-lock (ML) regime, harmonic modelocking and multiple pulsing [14-17]. In particular, it was shown in reference [17] that a large bistability domain exists between the $\mathrm{CW}$ and the ML operating regimes. The evolution of the number of pulses versus the pumping power was deeply investigated and it was demonstrated that the pulses were created and annihilated one by one and also that a large hysteresis occurred when the pump power was varied. In the mode-locked lasers based on the nonlinear polarization rotation, additional behaviours such as bound states or pulse fragmentation can be observed by simply rotating one of the phase plates used in the polarization controller set [1824]. Bound solitons have been also reported in figure eight fiber lasers [25,26]. It appears that multiple pulsing, bistability between the CW and the ML regimes, Q-switching and locked pulses are common features in fiber lasers independently from the exact optical configuration. In addition, most of these operating regimes can be obtained by a simple rotation of a phase plate in the cavity. 
From the theoretical point of view, the first models were based on master equations which had the advantage to be simple, but which did not allow to take into account the essential role play by the phase plates [27-31]. The models consisted in a phenomenological scalar equation assuming that all effects per pass were small. They included the groupvelocity dispersion (GVD), the optical Kerr nonlinearity, and a gain medium. The modelocking properties of a fiber laser can be accounted for through this approach for positive and negative GVD. The effects of the phase plates cannot be described in this way. Other approaches based on two coupled nonlinear equations have been also used [32,33]. We have developed intermediate models in which the vectorial nature of the electric field is taken into account but the final equation is scalar. The effects of the phase plates and the polarizer are explicitly included. In this paper, we give a comprehensive review of these models. The first one is a scalar model and was initially proposed to model the mode-locking properties of the ytterbium-doped ring fiber laser passively mode-locked through nonlinear polarization rotation [34]. It has been further extended to both negative GVD regime [35] and stretchedpulse regime [36]. This model includes the GVD, the birefringence, the optical Kerr nonlinearity and linear gain (i.e. gain saturation effects were neglected). The master equation is of complex cubic Ginzburg-Landau type and the coefficients take explicitly into account the orientation angles of the phase plates. This theoretical approach is presented in section II. In addition to the master equation, we discuss the stability of the continuous and mode-locked solutions as a function of the orientation of the phase plates in both normal and anomalous dispersion cases. The main advantage of this model is that the mode-locking solutions are known analytically and hence the stability of the ML solutions can be studied as a function of the orientation of the phase plates. In order to investigate the effect of the pumping power we decided to develop a second model including the gain saturation and preserving the inclusion of the phase plates. What we gained is all multistability phenomena and what we lost is that the model is not fully analytic. This model is presented in section III where multiple pulsing is investigated [37]. Its reduction to a quintic complex Ginzburg-Landau (CGL) equation is also demonstrated [38]. This is a very important result because the quintic CGL equation is often considered as a universal model supporting soliton solutions, using arbitrary coefficients, which are not related to physical nor experimental parameters [39,40]. We give here explicit relations between the coefficients of the equation and the physical parameters. Finally, we will consider the modelling of the passive harmonic mode-locking [41]. 


\section{II - A scalar model without saturation}

The aim of this section is to develop a theoretical model able to describe the mode-locking properties of a fiber laser passively mode-locked through nonlinear polarization rotation. The experimental setup generally includes an amplifying doped fiber, an optical isolator and a polarizer placed between two halfwave plates. Depending on the orientation of the phase plates, the laser can operate in CW, mode-locked, Q-switch or unstable regime [42]. For the modelling, we consider a birefringent rare-earth doped fiber in a unidirectional ring cavity with a fixed intracavity polarizer. The orientations of the eigenaxis of the fiber at each sides of the polarizer are taken as adjustable parameters (this is equivalent to consider two halfwave plates). The system under consideration is schematically represented in figure 1 .

\section{II - 1 - The master equation}

The starting point is the equations giving the evolution of the two electric field polarization components in a gain medium with Kerr nonlinearity and GVD. In the framework of the eigenaxis of the birefringent fiber moving at the group velocity, the pulse envelop evolution is described by the following system $[32,43]$

$$
\begin{aligned}
& i \frac{\partial u}{\partial z}-K u-\frac{\beta_{2}}{2} \frac{\partial^{2} u}{\partial t^{2}}+\gamma\left(u|u|^{2}+\left.\left.A u\right|_{v}\right|^{2}+B v^{2} u^{*}\right)=i g\left(1+\frac{1}{\omega_{g}^{2}} \frac{\partial^{2}}{\partial t^{2}}\right) u \\
& i \frac{\partial v}{\partial z}+K v-\frac{\beta_{2}}{2} \frac{\partial^{2} v}{\partial t^{2}}+\gamma\left(v|v|^{2}+A v|u|^{2}+B u^{2} v^{*}\right)=i g\left(1+\frac{1}{\omega_{g}^{2}} \frac{\partial^{2}}{\partial t^{2}}\right) v
\end{aligned}
$$

$\mathrm{K}=\pi\left(\mathrm{n}_{\mathrm{x}}-\mathrm{n}_{\mathrm{y}}\right) / \lambda\left(\mathrm{m}^{-1}\right)$ is the birefringent parameter, $\mathrm{n}_{\mathrm{x}, \mathrm{y}}$ are the refractive indexes along the $x$ and $y$ eigenaxis and $\lambda$ is the optical wavelength. $\beta_{2}\left(\mathrm{ps}^{2} / \mathrm{m}\right)$ is the GVD coefficient. $\gamma=2 \pi \mathrm{n}_{2} /\left(\lambda \mathrm{A}_{\text {eff }}\right)$ is the nonlinear coefficient, $\mathrm{n}_{2}\left(\mathrm{~m}^{2} / \mathrm{W}\right)$ is the nonlinear index coefficient and $\mathrm{A}_{\text {eff }}\left(\mathrm{m}^{2}\right)$ the effective core area of the fiber. $\mathrm{A}$ and $\mathrm{B}$ are the dielectric coefficients. In isotropic media $A=2 / 3$ and $B=1 / 3$ [43]. $\mathrm{g}\left(\mathrm{m}^{-1}\right)$ is the gain coefficient and $\omega_{\mathrm{g}}=2 \pi \mathrm{c} \Delta \lambda / \lambda^{2}\left(\mathrm{ps}^{-1}\right)$ is the spectral gain bandwidth.

We report here the outline of the derivation of the master equation only; the full detail is given in [34]. The propagation problem (1-2) is first solved using a first order perturbative 
procedure assuming that $\beta_{2}, \gamma$ and the gain filtering $\rho=\mathrm{g} / \omega_{\mathrm{g}}^{2}$ are small over one round-trip of the cavity. Then the effects of the phase plates and of the polarizer are determined, which yields the electric field amplitude $f_{n}$ just after the polarizer. There it has a well-defined linear polarization parallel to the passing axis of the polarizer. Thanks to the perturbative solutions, it is possible to express the electric field amplitude at the $(n+1)^{\text {th }}$ round-trip $f_{n+1}$ as a function of its amplitude at the $n^{\text {th }}$ round-trip. Finally, a multiscale analysis [44] allows to obtain a continuous equation. The resulting equation for the electric field amplitude $\mathcal{F}$ is of the cubic complex Ginzburg-Landau (CGL) type and, for a large number of round trips, is written as

$$
\mathrm{i} \frac{\partial \mathcal{F}}{\partial \mathrm{z}}=\mathrm{ig}_{1} \mathcal{F}+\left(\frac{\beta_{2}}{2}+\mathrm{i} \rho\right) \frac{\partial^{2} \mathcal{F}}{\partial \mathrm{t}^{2}}+\left(\mathcal{D}_{\mathrm{r}}+\mathrm{i} \mathcal{D}_{\mathrm{i}}\right) \mathcal{F}|\mathcal{F}|^{2}
$$

The gain filtering writes now $\rho=\mathrm{g}_{0} / \omega_{\mathrm{g}}^{2}$, where $\mathrm{g}_{0}$ is the gain coefficient which compensates the linear losses:

$$
\mathrm{g}_{0}=-\frac{1}{2 \mathrm{~L}} \ln \left(\beta^{2}|Q|^{2}\right)
$$

$\beta$ is the transmission coefficient of the polarizer (it takes into account the output coupling which has not yet been included) and the parameter $Q$ is

$$
Q=\cos \left(\theta_{+}-\theta_{-}\right) \cos \mathrm{KL}-\mathrm{i} \cos \left(\theta_{+}+\theta_{-}\right) \sin \mathrm{KL} .
$$

$\theta_{-}$and $\theta_{+}$are the angles between one eigenaxis of the fiber and the passing axis of the polarizer before and after it, respectively (see figure 1). $\mathrm{g}_{1}$ is the excess of linear gain and it is still a free parameter.

The effective nonlinear self-phase modulation $\mathcal{D}_{\mathrm{r}}$ and effective nonlinear gain (or absorption) $\mathcal{D}_{\mathrm{i}}$ are the real and imaginary parts of $\mathcal{D}=-\mathcal{P} /(Q \mathrm{~L})$ where 


$$
\begin{aligned}
\mathcal{P}=\gamma\left[\frac{\mathrm{e}^{2 \mathrm{~g}_{0} \mathrm{~L}}-1}{2 \mathrm{~g}_{0}}\left(Q+\frac{\mathrm{A}-1}{2} \sin 2 \theta_{+}\left(\sin \left(\theta_{+}+\theta_{-}\right) \cos \mathrm{KL}-\mathrm{i} \sin \left(\theta_{+}-\theta_{-}\right) \sin \mathrm{KL}\right)\right)\right. \\
\left.+\frac{\mathrm{B}}{2} \sin 2 \theta_{+}\left(\sin \theta_{+} \cos \theta_{-} \mathrm{e}^{-\mathrm{iKL}} \frac{\mathrm{e}^{2 \mathrm{~g}_{0} \mathrm{~L}+4 \mathrm{iKL}}-1}{2 \mathrm{~g}_{0}+4 \mathrm{iK}}+\cos \theta_{+} \sin \theta_{-} \mathrm{e}^{\mathrm{iKL}} \frac{\mathrm{e}^{2 \mathrm{~g}_{0} \mathrm{~L}-4 \mathrm{iKL}}-1}{2 \mathrm{~g}_{0}-4 \mathrm{iK}}\right)\right]
\end{aligned}
$$

Equation (3) was first proposed by K.P.Komarov for the description of passively mode-locked solid-sate lasers $[45,46]$ and is formally identical to the fiber laser master equation further proposed by Haus [27]. However, the coefficients of (3) explicitly depend on the orientation of the eigenaxis of the fiber at both sides of the polarizer. An essential feature is the arising of an effective nonlinear gain or absorption $\mathcal{D}_{\mathrm{i}}$ that results from the combined effects of the nonlinear polarization rotation, the losses due to the polarizer, and the linear gain. The value and the sign of $\mathcal{D}_{\mathrm{i}}$ depend on the angles $\theta_{+}$and $\theta_{-}$. At this stage, it is important to stress that, in contrast with previous approaches based on phenomenological demonstration of the cubic CGL equation, all the coefficients appearing in the master equation (3) depend explicitly on real physical parameters.

\section{II - 2 - Normal dispersion case}

We consider in this section the normal dispersion case corresponding to $\beta_{2}>0$. Our objective is to investigate the stability of the $\mathrm{CW}$ and the mode-lock solutions versus the orientation angles $\theta_{+}$and $\theta_{-}$. Recall that a variation of theses angles is equivalent to a rotation of the halfwave plates placed generally at each side of the polarizer. For the numerical simulations we use the following values for the parameters: $\lambda=1.08 \mu \mathrm{m}$, $\mathrm{K}=1.5 \mathrm{~m}^{-1}, \beta_{2}=0.026 \mathrm{ps}^{2} / \mathrm{m}, \mathrm{L}=9 \mathrm{~m}, \gamma=3 \cdot 10^{-3} \mathrm{~W}^{-1} \mathrm{~m}^{-1}$ and $\omega_{\mathrm{g}}=10^{13} \mathrm{~s}^{-1}$. These values are well adapted to the real case of the ytterbium-doped fiber laser [34].

Let us first consider the nonzero stationary solution of the CGL equation (3) characterized by a constant modulus. If we admit that the excess of linear gain $\mathrm{g}_{1}$ will selfadjust to a value for which a stable solution exists, the stability of the constant solution depends on the sign of the effective nonlinear gain $\mathcal{D}_{\mathrm{i}}$. It is stable when $\mathcal{D}_{\mathrm{i}}<0$ only [34].

We expect to observe continuous laser emission when the constant solution is stable, and only in this case.

A localized analytical solution of the CGL equation (3) can also be written [34, 47]. Qualitatively, localized pulse formation can be expected when the excess of linear gain $\mathrm{g}_{1}$ is 
negative and the nonlinear gain $\mathcal{D}_{\mathrm{i}}$ is positive, as it is suggested in figure 2 . The effective self-phase modulation $\mathcal{D}_{\mathrm{r}}$ is always negative, and the dispersion $\beta_{2}$ is positive. Therefore their conjugated effect leads to the increase of the pulse width. If $g_{1}$ is positive and $\mathcal{D}_{\mathrm{i}}$ negative (figure $2 \mathrm{a}$ ), the nonconservative effects decrease the amplitude of the top of the pulse, and increase it at the bottom. As a consequence, no stable localized pulse can be formed. If in the contrary $g_{1}$ is negative and $\mathcal{D}_{\mathrm{i}}>0$ (figure $2 \mathrm{~b}$ ), energy appears at the top of the pulse and disappears at its bottom, yielding some pulse narrowing, which could be expected to balance the broadening caused by the nonlinear index variations. On these grounds, the exact solution corresponds to a completely unstable pulse when $\mathrm{g}_{1}>0$ and $\mathcal{D}_{\mathrm{i}}<0$ (the solution itself is unstable in this case since other pulses arise spontaneously from the background) and to a stable pulse when $\mathrm{g}_{1}<0$ and $\mathcal{D}_{\mathrm{i}}>0$.

Results can be conveniently summarized in a stability diagram in the plane $\left(\theta_{+}, \theta_{-}\right)$ which gives the regions of stability of the different solutions. Such cartography is given in figure 3. The domain in dark grey corresponds to stable mode-locked operation. The white domain corresponds to stable $\mathrm{CW}$ regime and finally, in the light grey region neither stable localized solution nor constant one exist. The laser is expected to be unstable in this region, experimentally this instability can be either a Q-switch regime or an imperfect mode-locking or a mixture of Q-switch and mode-locked [42]. The periodicity is $180^{\circ}$ for both angles $\theta_{+}$ and $\theta_{-}$, as it could be expected. At first glance, the period seems to be only $90^{\circ}$ versus $\theta_{+}$. However, it can be demonstrated that the effective nonlinear gain is different in the region $\theta_{+} \in\left[0,90^{\circ}\right]$ and in the region $\theta_{+} \in\left[90^{\circ}, 180^{\circ}\right]$ [34]. Hence these two regions are not equivalent and the periodicity is $180^{\circ}$. Finally, it is worth to note that the resulting diagram is in very good agreement with experimental data obtained with an ytterbium-doped double-clad fiber laser operating at $1.08 \mu \mathrm{m}[34,48]$.

\section{II - 3 - Anomalous dispersion case}

We consider in the section the anomalous dispersion case obtained when $\beta_{2}<0$. The change of the sign of the chromatic dispersion has strong structural consequences. Indeed, in the present case, the explicit localized solutions of the master equation can be effectively stable. Therefore, it is possible to fully characterize the pulses, which is not the case for positive GVD. The master equation remains the same as used in the previous section for positive GVD. For the numerical simulations we use the following values of the parameters: $\lambda=1.55 \mu \mathrm{m}$, 
$\mathrm{K}=0.1 \mathrm{~m}^{-1}, \beta_{2}=-0.002 \mathrm{ps}^{2} / \mathrm{m}, \mathrm{L}=9 \mathrm{~m}, \gamma=2 \cdot 10^{-3} \mathrm{~W}^{-1} \mathrm{~m}^{-1}$ and $\omega_{\mathrm{g}}=1.57 \cdot 10^{13} \mathrm{~s}^{-1}$ (or equivalently $\Delta \lambda=30 \mathrm{~nm}$ ).

We first consider the nonzero stationary solution of (3). Neither the solution nor the analysis of its stability depend on the sign of the chromatic dispersion. Thus, results of previous section are still valid here. The constant solution is stable when $\mathcal{D}_{\mathrm{i}}<0$ only and, continuous laser emission occurs if this condition is fulfilled.

Let us now investigate the localized solutions of (3) describing short pulses. In order to use the results of Akhmediev et al. [49,50], it is convenient to write the CGL equation (3) in the normalized form

$$
\mathrm{i} \frac{\partial \psi}{\partial \mathrm{z}}+\frac{1}{2} \frac{\partial^{2} \psi}{\partial \tau^{2}}+\psi|\psi|^{2}=\mathrm{ig}_{1} \psi+\mathrm{iD} \psi|\psi|^{2}+\mathrm{iR} \frac{\partial^{2} \psi}{\partial \tau^{2}}
$$

where $\psi=\sqrt{\left|\mathcal{D}_{\mathrm{r}}\right|} \mathcal{F}, \tau=\mathrm{t} / \sqrt{\left|\beta_{2}\right|}, \mathrm{R}=\rho /\left|\beta_{2}\right|$ and $\mathrm{D}=-\mathcal{D}_{\mathrm{i}} / \mathcal{D}_{\mathrm{r}}$.

Equation (7) admits an explicit localized solution which can be found in [35,49,50]. In the case of positive nonlinear gain $\mathrm{D}$ or $\mathcal{D}_{\mathrm{i}}$, Akhmediev et al. $[49,50]$ have shown that the localized solution is stable when the zero solution is unstable, thus when the excess of linear gain $g_{1}$ is positive. In the plane $(R, D)$, the solution is stable below the curve

$$
\mathrm{D}=\mathrm{D}_{\mathrm{s}}=\mathrm{R} \frac{3 \sqrt{1+4 \mathrm{R}^{2}}-1}{4+18 \mathrm{R}^{2}}
$$

and unstable above this curve. When the nonlinear gain $\mathcal{D}_{\mathrm{i}}$ is negative, no such condition is known. The nonzero constant solution is stable in this case for a positive excess of linear gain $\mathrm{g}_{1}$, allowing to conclude to $\mathrm{CW}$ laser emission. However, a situation when bistability between continuous and mode-locked regime occurs cannot be ruled out. This will mean that the mode-locking would not be self-starting. These problems of multistability will be addressed in the next section with the second model including the saturation of the gain.

Figure 4 summarizes the stability of the solutions in the plane $(\mathrm{R}, \mathrm{D})$. The results can be also represented in the plane $\left(\theta_{+}, \theta_{-}\right)$limited to $180^{\circ} \times 180^{\circ}$ because of the periodicity of the solutions (figure 5). The white regions correspond to stable pulses and then to stable self- 
starting mode-locking because the stationary solution is unstable. In the grey domains the stationary solution is stable and the laser spontaneously operates in $\mathrm{CW}$ regime. However, because we have no information about the stability of the pulses in these regions, the laser also might operate in mode-locking regime if a perturbation is applied. In the hatched regions, both solutions are unstable and the laser is expected to operate in chaotic or Q-switch regime.

At this stage, significant differences appear in comparison with the normal dispersion case. Indeed, the domains of self-starting mode-locking are considerably reduced. In addition, bistability between the continuous and mode-locked regime is not excluded in the present case, while no bistability has been observed in the normal dispersion regime.

Equation (3) does not take into account the gain saturation. However it can be introduced as an external condition allowing to fully characterize the pulse. We first compute the energy of the pulses as a function of the orientation angles $\left(\theta_{+}, \theta_{-}\right)$. At first order, the saturated gain of the medium is

$$
\mathrm{g}_{0}=\frac{\mathrm{g}^{\prime}}{1+\frac{\mathrm{E}}{\mathrm{W}_{\mathrm{s}}}},
$$

where $\mathrm{g}^{\prime}$ is the unsaturated gain, $\mathrm{W}_{\mathrm{s}}$ the saturation energy, and $\mathrm{E}$ the pulse energy. Relation (9) allows to extract the pulse energy provided that the threshold $g_{0}$ is known, which is the case (see equation (4)). For the numerical simulation, we take $\mathrm{g}^{\prime}=1.26 \mathrm{~m}^{-1}$ and $\mathrm{W}_{\mathrm{s}}=0.1 \mathrm{pJ}$ [35]. The results are presented in figure 6 which gives the repartition of the energy $\mathrm{E}$ in the plane $\left(\theta_{+}, \theta_{-}\right)$. The energy strongly varies with $\theta_{+}$and $\theta_{-}$. The most energetic pulses are obtained in the vicinity of $\left(0^{\circ}, 0^{\circ}\right)$ and $\left(90^{\circ}, 90^{\circ}\right)$. In these regions the energy is about $10 \mathrm{pJ}$ and is very close to the one reported by Haus et al. [51].

The pulse duration is also an important characteristic of the mode-locked laser. In our model, the pulse duration is obtained analytically, and depends on the orientation angles $\theta_{+}$ and $\theta_{-}$. It takes the form [35]

$$
\mathrm{t}_{0}=\frac{2\left|\beta_{2}\right| \mathrm{N}^{2}}{\mid \mathcal{D}_{\mathrm{r}}\left(\frac{\mathrm{g}^{\prime}}{\mathrm{g}_{0}}-1\right) \mathrm{W}_{\mathrm{s}}},
$$


where

$$
N=\frac{\sqrt{3\left(\sqrt{9(1+2 D R)^{2}+8(D-2 R)^{2}}-3(1+2 D R)\right)\left(1+4 R^{2}\right)}}{2|D-2 R|} .
$$

Figure 7 gives the variations of the pulse duration $t_{0}$ versus $\theta_{+}$and $\theta_{-}$. As the pulse energy, the duration undergoes large variations. Only small regions lead to ultrashort pulses. The shortest pulse width obtained with the model is about $90 \mathrm{fs}$. This lowest value depends on the spectral gain bandwidth, which plays an important role since it can directly limit the pulse duration. Beyond $\Delta \lambda \approx 15 \mathrm{~nm}$ the pulse duration does not change when the spectral gain bandwidth is varied. In this case, the limitation is due to the GVD. Below $\Delta \lambda \approx 15 \mathrm{~nm}$ the pulse width increases when $\Delta \lambda$ decreases, the pulse duration is then limited by the spectral gain bandwidth. It is worth to note that the shortest pulses are obtained for the same values of the angles $\theta_{+}$and $\theta_{-}$which maximize the pulse energy. Both parameters can be optimized simultaneously.

\section{II - 4 - Summary}

The main advantages of the analysis are that it is fully analytic and that it takes into account explicitly the phase plates. This allows to easily investigate the stability of the mode-locked solution as a function of the orientation of the intracavity phase plates. The same theoretical approach has been used for different laser configurations: the ytterbium-doped fiber laser operating in the normal dispersion regime [34], the erbium-doped fiber laser operating in the anomalous dispersion regime [35], the stretched-pulse erbium-doped fiber laser [36], the ytterbium-doped fiber laser in a configuration involving two sets of a halfwave and quarterwave plates [52]. Although the agreement with experimental data is very good for some laser configurations and for different operating regimes, some differences still exist with experiment and the model does not give any account of several experimental features such as hysteresis versus the pump power or versus the orientation of the phase plates. This approach has intrinsically two weaknesses. First it is an average approach in that sense that all the effects are averaged over several round-trips of the cavity. The consequence is that the resulting model fails to correctly describe the mode-locking regime in a stretched-pulse 
configuration where the pulse undergoes large variations over a cavity round-trip [48]. This problem can be overcome by a full numerical simulation of the field evolution inside the cavity [32,33,53-55]. The second weakness is that the above analysis ignores the gain saturation and cannot predict multistability phenomena neither the multiple pulsing behaviours. This problem can be solved by the inclusion of the saturation. This is the subject of the following section which presents a scalar model with saturation which was motivated by the weaknesses discussed above.

\section{III - A scalar model with saturation}

The initial goal of this second approach was to develop a simple theoretical model allowing to describe the dynamics of multiple pulsing in a passively mode-locked fiber laser. Hysteresis phenomena versus the pump power or versus the orientation of the phase plates have been correctly modelled in the same way $[37,56]$. Beyond the initial results, the model has allowed to investigate new interesting and important features. The first one was the possibility of deriving for the first time quintic complex Ginzburg-Landau equation from the basic equations modelling ring fiber lasers [38]. The second is the modelling of other behaviours such as Q-switching [56] or passive harmonic mode-locking [41], with a suitable adaptation of the initial model.

\section{III - 1 - The model}

We consider a rare-earth doped fiber ring laser passively mode-locked through nonlinear polarization rotation. In contrast with the previous section we will neglect the linear birefringence of the fibers. The setup is schematically represented in figure 8. For isotropic fibers this scheme involves all necessary elements for controlling the nonlinear losses. After the polarizing isolator the electric field has a linear polarization. Such state of polarization does not experience polarization rotation in the fiber because the rotation angle is proportional to the area of the polarization ellipse. Consequently, it is necessary to place a quarterwave plate $3\left(\alpha_{3}\right.$ represents the orientation angle of one eigenaxis of the plate with respect to the laboratory frame). At the output of the fiber, the direction of the elliptical polarization of the central part of the pulse can be rotated towards the passing axis of the polarizer by the halfwave plate 2 (the orientation angle is $\alpha_{2}$ ). Then this elliptical polarization can be transformed into a linear one by the quarterwave plate 1 (the orientation angle is $\alpha_{1}$ ). In this situation the losses for the central part of the pulse are minimum while the wings undergo 
strong losses. The setup of figure 8 has been modelled as follows [37]. The fiber has been assumed to have group velocity dispersion (GVD), optical Kerr nonlinearity and saturable gain. The nonlinear losses are computed as follows: we consider the propagation equations for the two components of the electric field, taking into account the Kerr nonlinearity (self- and cross-phase modulation terms were considered together with four-wave mixing terms [57]), but neglecting dispersion. These equations can be solved analytically, and then the three phase plates and the polarizer are taken into account. On the other hand, a scalar equation has been written for a wave propagating in a saturable amplifying medium with GVD, to account for dispersion and gain. The resulting model assumes localized effect for the nonlinear loss due to the Kerr nonlinearity and the phase plates, while gain and GVD are distributed. In dimensionless form, the final set of equations for the electric field amplitude is [37]

$$
\begin{gathered}
\frac{\partial E}{\partial \zeta}=\left(D_{r}+\mathrm{iD}_{\mathrm{i}}\right) \frac{\partial^{2} \mathrm{E}}{\partial \tau^{2}}+\left(\mathrm{G}+\mathrm{i}|\mathrm{E}|^{2}\right) \mathrm{E} \\
\mathrm{E}_{\mathrm{n}+1}(\tau)=-\beta\left[\cos \left(\mathrm{pI}_{\mathrm{n}}+\alpha\right) \cos \left(\alpha_{1}-\alpha_{3}\right)+\mathrm{i} \sin \left(\mathrm{pI}_{\mathrm{n}}+\alpha\right) \sin \left(\alpha_{1}+\alpha_{3}\right)\right] \mathrm{E}_{\mathrm{n}}(\tau),
\end{gathered}
$$

where $\zeta=\mathrm{z} / \mathrm{L}, \tau=\mathrm{t} / \delta \mathrm{t}, \mathrm{G}=\mathrm{a} /\left(1+\mathrm{b} \int \mathrm{Id} \tau\right), \mathrm{D}_{\mathrm{r}}=\mathrm{GD}_{\mathrm{r}}^{0}+\mathrm{d}_{\mathrm{r}}, \mathrm{D}_{\mathrm{r}}^{0}=2 /\left(\left|\beta_{2}\right| \mathrm{L} \omega_{\mathrm{g}}^{2}\right), \mathrm{I}_{\mathrm{n}}=\left|\mathrm{E}_{\mathrm{n}}\right|^{2}$, $d_{r}=2 \rho_{c} /\left|\beta_{2}\right|, \quad p=B \sin 2 \alpha_{3}, \quad a=g_{0} L, \quad b=I_{r} \delta t /\left(P_{s a t} T_{a}\right), \quad \delta t=\sqrt{\left|\beta_{2}\right| L / 2}, \quad I_{r}=1 / \gamma L$, $\alpha=2 \alpha_{2}-\alpha_{1}-\alpha_{3}, \delta \mathrm{t}=\sqrt{\beta_{2} \mathrm{~L} / 2}$.

$\gamma\left(\mathrm{W}^{-1} \mathrm{~m}^{-1}\right)$ is the nonlinear coefficient related to the nonlinear index coefficient $\mathrm{n}_{2}$, and $\mathrm{B}=1 / 3$ for silicate fibers [43]. $\beta$ is the transmission coefficient of the polarizer (free parameter). $\beta_{2}\left(\mathrm{ps}^{2} \mathrm{~m}^{-1}\right)$ is the second order GVD. $\mathrm{T}_{\mathrm{a}}=\mathrm{Ln}_{0} / \mathrm{c}(\mathrm{s})$ is the photon round trip time, $\mathrm{n}_{0}$ the refractive index, $\mathrm{c}$ the velocity of light in free space, $\mathrm{g}_{0}\left(\mathrm{~m}^{-1}\right)$ the unsaturated gain, and $\mathrm{P}_{\mathrm{sat}}(\mathrm{W})$ the saturating power. The dimensionless parameter a represents the pumping.

$\mathrm{P}_{\mathrm{sat}}=\left(\mathrm{h} v \pi \mathrm{r}^{2}\right) /\left(\sigma \mathrm{T}_{1}\right)$, where $\mathrm{h} v(\mathrm{~J})$ is the photon energy, $\sigma\left(\mathrm{m}^{2}\right)$ the stimulated emission cross-section, $\mathrm{T}_{1}(\mathrm{~s})$ the lifetime of the upper level of the lasing transition, and $\mathrm{r}(\mathrm{m})$ the radius of the fiber core, $v$ is the optical frequency and $h$ the Planck's constant. $\omega_{g}\left(s^{-1}\right)$ is the spectral gain bandwidth. $\rho_{c}$ describes the frequency-dependent loss due to both additional 
spectrally selective elements for control of a radiation spectrum or uncontrolled spectrally selective losses related with intracavity elements. $D_{i}$ and $L(m)$ are the dispersion and the length of the fiber, respectively.

The numerical procedure starts from the evaluation of the electric field after passing through the Kerr medium, the phase plates and the polarizer, using equation (13). The resulting electric field is then used as the input field to solve equation (12) over a distance L, using a standard split-step Fourier algorithm. The computed output field is used as the new input for equation (13). This iterative procedure is repeated until a steady-state is achieved.

For the numerical simulations we consider the practical case of an ytterbium-doped fiber laser. The values of the different parameters are $\gamma=3 \cdot 10^{-3} \mathrm{~W}^{-1} \mathrm{~m}^{-1}$, $\beta_{2}=0.026 \mathrm{ps}^{2} / \mathrm{m}, \quad \mathrm{L}=9 \mathrm{~m}, \omega_{\mathrm{g}}=10^{13} \mathrm{~s}^{-1}, \quad \mathrm{r}=9 \cdot 10^{-6} \mathrm{~m}, \quad \sigma=2.5 \cdot 10^{-24} \mathrm{~m}^{2} \quad[58]$ $\mathrm{T}_{1}=8 \cdot 10^{-4} \mathrm{~s}[58]$ and $\beta=0.95$.

\section{III - 2 - Multiple pulsing and hysteresis phenomena}

In this section we are interested in multiple pulse operation of the laser and in the dynamics versus the pumping parameter. In the framework of our model, multiple pulsing requires additional spectrally selective losses, i.e. $d_{r} \neq 0$ [37]. Figure 9 shows an example of multiple pulses consisting in a bunch of three pulses. The pumping parameter is $\mathrm{a}=1.2$. At this stage, it is interesting to investigate the evolution of the operating regime when the pumping parameter is varied. It is convenient to represent the results in a diagram which gives the number $\mathrm{N}$ of pulses for increasing and decreasing values of the pumping parameter a . Results of the simulations are given in figure 10 in the normal dispersion regime. The pump parameter at lasing threshold is $\mathrm{a}_{\text {th }}=0.68$. Several interesting features can be seen in this diagram. Increasing the pumping, the laser is first continuous and then directly falls into multiple pulsing regime $(\mathrm{N}=3)$ for a pump parameter $\mathrm{a}_{\mathrm{ML}}=2.1$. If the pumping parameter is further increased, the number of pulses increases. The dynamics is different if the pump is decreased. Indeed, the number of pulses disappears one by one for particular values of a. This occurs until the laser becomes continuous again, for a value of the pumping parameter close to its threshold value. Moreover, when the laser is in the N-pulse mode-locked regime, the values of the pumping parameter for which one additional pulse appears by increasing a is different from those obtained when a is decreased. Hence the formation and annihilation of each pulse shows pump power hysteresis. The more pulses exist in the cavity, the bigger the 
hysteresis. Similar results are obtained in the anomalous dispersion regime. Our theoretical results are in very good agreement with experimental data obtained in passively mode-locked fiber lasers $[2-5,11,12,17]$.

Let us now consider the effects of the rotation of the phase plates on the operating regime of the fiber laser. It is well established that large hysteresis occurs in passively modelocked fiber lasers when one phase plate is rotated. In particular, bistability between the CW and the mode-locked regime is commonly observed [17,48]. In the framework of the model based on equations (12-13), numerical integrations allow to point out bistability behaviours and hysteresis phenomena. Results are given in figure 11 which gives the evolution of the operating regime as a function of the angle $\alpha$. The orientations of the phase plates number 1 and 3 are fixed and the halfwave plate 2 is rotated in the right handed and then in the left handed direction. Results of figure 11 show that the laser is first CW and then switches towards the mode-locked regime for $\alpha=\alpha_{\mathrm{ML}}$. At this stage, if the phase plate is rotated back in the opposite direction, the laser becomes again $\mathrm{CW}$ but for a lower value of $\alpha$. A large bistability domain is then obtained versus the orientation angle of the phase plates. In addition, when the laser is in the ML regime, the number $\mathrm{N}$ of pulses increases when the phase plate is rotated, pulses appear one by one. Again, large hysteresis is obtained versus the orientation angle. These results are due to the variations of the nonlinear losses resulting from a rotation of a phase plate.

It is well known that solid-state lasers with saturable absorbers can deliver undamped spikes (Q-switch regime) and fiber lasers are not an exception because of the fast saturable absorption resulting from the nonlinear losses. Indeed, this is usually observed experimentally in rare-earth doped fiber laser passively mode-locked $[4,5,42]$. To model these regimes it is necessary to take into account the finiteness of the relaxation time of the gain medium $\tau_{\mathrm{g}}$. This is achieved by considering the evolution equation of the gain $\mathrm{G}$. The laser equations become

$$
\begin{gathered}
\frac{\partial \mathrm{E}}{\partial \zeta}=\left(\mathrm{D}_{\mathrm{r}}+\mathrm{iD}_{\mathrm{i}}\right) \frac{\partial^{2} \mathrm{E}}{\partial \tau^{2}}+\left(\mathrm{G}+\mathrm{i}|\mathrm{E}|^{2}\right) \mathrm{E} \\
\mathrm{E}_{\mathrm{n}+1}(\tau)=-\beta\left[\cos \left(\mathrm{pI}_{\mathrm{n}}+\alpha\right) \cos \left(\alpha_{1}-\alpha_{3}\right)+\mathrm{i} \sin \left(\mathrm{pI} \mathrm{I}_{\mathrm{n}}+\alpha\right) \sin \left(\alpha_{1}+\alpha_{3}\right)\right] \mathrm{E}_{\mathrm{n}}(\tau) \\
\tau_{\mathrm{g}} \frac{\partial \mathrm{G}}{\partial \zeta}+(\mathrm{G}-\mathrm{a})=-\mathrm{Gb} \int \mathrm{Id} \tau
\end{gathered}
$$


It can be shown that, under some approximations, the system of equations (14-16) can be reduced to the usual equations of a laser with a saturable absorber [56]. Therefore, Qswitching can be expected from these equations. This is confirmed in figure 12 which represents the evolution of the integrated intensity $J=\int \mathrm{Id} \tau$ versus the round-trip number $\zeta$. The difference between figures $12(a)$ and $12(b)$ is only on the initial data. The hysteresis behaviour versus the pumping parameter is demonstrated in figure 13 . When the laser is turned on, it is first continuous and then switches towards a Q-switch regime. The switching values of the pumping parameter differ depending on whether the pump is increased or decreased. This bistable behaviour is in very good agreement with the experimental results reported in [42], which concern the ytterbium-doped double-clad fiber laser. For the range of pumping rates investigated, the mode-locked regime is also stable, as shown in figure 13. However, it is not self-starting, since there is no switch from the CW or Q-switch regimes.

\section{III - 3 - Reduction to a quintic complex Ginzburg-Landau equation}

Complex Quintic Ginzburg-Landau (CGLQ) equation is a universal model commonly used to describe or predict specific fiber laser operating regimes, such as bound states [39,40,47,5961]. In any case, the coefficients of the equation are given in a purely phenomenological way. Therefore, it was of great importance to derive the CGLQ equation from basic principles in order to obtain the relation between the coefficients of the equation and the experimental parameters. We have established the CGLQ equation in [38] starting from the model developed in [37]. The optical configuration is given in figure 8. The procedure used is the following. The discrete sequence (13) is first interpolated by a continuous function $f$ leading to a differential equation accounting for the nonlinear effects. The equation (12) is rewritten with the gain saturation neglected. Finally, considering the weak amplitude approximation, the nonlinear term of the resulting equation is expanded in a power series of $|f|^{2}$. In dimensionless form, we obtain the CGLQ equation $[38,47,59]$

$$
\mathrm{i} \frac{\partial \psi}{\partial \zeta}+\frac{\mathrm{D}}{2} \frac{\partial^{2} \psi}{\partial \tau^{2}}+\psi|\psi|^{2}=\mathrm{i} \delta \psi+\mathrm{i} \eta \frac{\partial^{2} \psi}{\partial \tau^{2}}+\mathrm{i} \varepsilon \psi|\psi|^{2}+\mathrm{i} \mu \psi|\psi|^{4}
$$

with

$$
\psi=\sqrt{\gamma \mathrm{L}} f, \zeta=\mathrm{z} / \mathrm{L}, \tau=\mathrm{t} /\left(\sqrt{\left|\beta_{2}\right|} \mathrm{L}\right), \mathrm{D}=-\operatorname{sgn} \beta_{2}, \eta=\mathrm{g}_{0} /\left(\omega_{\mathrm{g}}^{2}\left|\beta_{2}\right|\right),
$$




$$
\varepsilon=-\mathrm{B} \sin 2 \alpha_{2} \tan \alpha, \delta=\operatorname{Lg}_{0}+\ln \left|\beta \cos 2 \alpha_{3} \cos \alpha\right|, \mu=-\mathrm{B}^{2} \frac{\sin ^{2} 2 \alpha_{3}}{2 \cos ^{2} \alpha}
$$

An effective quintic absorption-gain term has been derived this way, with the nonvanishing coefficient $\mu$, without any quintic nonlinearity of the material. Thus the CGLQ equation can be valid even if the quintic nonlinearity is absent. Note that the effective quintic nonlinear index is zero in the approximation considered. Relations (18) and (19) make the link between the physical parameters, essentially the orientation angles of the phase plates, and the coefficients of the CGLQ equation. The set of coefficients of the CGLQ is very wide and can very hardly be investigated systematically by numerical methods. The coefficients given above, may give an orientation for further studies. For illustrative purpose, we give in figure 14 an example of a bound state of three pulses which has been calculated for the physical parameters $\quad \mathrm{L} \beta_{2}=-0.05 \mathrm{ps}^{2} \quad, \quad \beta=0.95 \quad, \quad \mathrm{~g}_{0} \mathrm{~L}=2.5 \quad, \quad \omega_{\mathrm{g}}=10 \mathrm{ps}^{-1} \quad$ and $\left(\alpha, \alpha_{3}\right)=\left(-85.047^{\circ}, 2.985^{\circ}\right)$.

\section{III - 4 - Passive harmonic mode-locking}

Multiple pulsing is a very usual behaviour in passively mode-locked fiber lasers independently of the exact optical configuration. Generally the pulses are randomly spaced in the laser cavity leading to the so-called bunch states $[5,7,14,17]$. To arrange the pulses within the cavity, active techniques can be used, as amplitude or phase modulation [62,63]. However, this arrangement and the realization of harmonic passive mode-locking can arise spontaneously without any active control [64,65]. Investigation of mechanisms of pulse ordering in the cavity is of great importance for the control of high repetition rate fiber laser operation. We have developed a simple theoretical model for both active and passive harmonic mode-locking [41]. Again, we consider a fiber laser passively mode-locked by means of the nonlinear polarization rotation (figure 8). The model is very similar to the one presented in section III - 1 except that we introduce an additional equation to account for the mechanism of pulse ordering. The latter can be either the depletion and relaxation of the gain, or an inertial nonlinear refractive index with finite relaxation time. The normalized equations for the electric field have the form

$$
\frac{\partial E}{\partial \zeta}=\left(D_{r}+i D_{i}\right) \frac{\partial^{2} E}{\partial \tau^{2}}+\left(G+X-\langle X\rangle+i q|E|^{2}\right) E
$$




$$
\mathrm{E}_{\mathrm{n}+1}(\tau)=-\beta\left[\cos \left(\mathrm{pI}_{\mathrm{n}}+\alpha\right) \cos \left(\alpha_{1}-\alpha_{3}\right)+\mathrm{i} \sin \left(\mathrm{pI}_{\mathrm{n}}+\alpha\right) \sin \left(\alpha_{1}+\alpha_{3}\right)\right] \mathrm{E}_{\mathrm{n}}(\tau)
$$

All parameters have been defined in the previous sections, except $\mathrm{q}$ which is the normalized Kerr nonlinearity, and $\mathrm{X}$ which describes the specific mechanism of pulse ordering: passive modulation of the net gain or the refractive index. $\langle X\rangle$ is the average value of $X$ over one round-trip of the cavity.

The passive modulation is taken into account using the evolution equation

$$
\tau_{\mathrm{r}} \frac{\partial \mathrm{X}}{\partial \tau}+(\mathrm{X}-\mathrm{h} \tilde{a})=-\mathrm{X} \tilde{\mathrm{b}} \mathrm{I}
$$

where $\tau_{\mathrm{r}}$ is the relaxation time, $\tilde{\mathrm{b}}$ is the saturation parameter, and $\mathrm{X}$ represents the gain or the refractive index. The numerical procedure is as follows. At each step for $\zeta$, knowing $\mathrm{I}(\tau)$, we obtain $X(\tau)$ from (22). Then from $X(\tau)$ we determine its average value over one roundtrip $\langle X\rangle$. Finally we deduce the modulation term $X(\tau)-\langle X\rangle$ introduced in equation (20).

For $\mathrm{h}=\mathrm{i}$, equation (22) describes the inertial refractive index. If $\mathrm{h}=1$ and $\tau_{\mathrm{r}}, \tilde{\mathrm{a}}, \tilde{\mathrm{b}}$ correspond to the parameters of the gain medium, then (22) describes the evolution of the gain. In addition if $\mathrm{h}=1$ and $\tilde{\mathrm{a}}<0$, equation (22) accounts for a saturable absorber, if $\tilde{\mathrm{a}}>0$ for a darkening absorber. The latter can be, for instance, a reverse saturable absorber which exhibits an increasing absorption versus the intensity [66]. Immediately after the ultrashort pulse, the characteristic $X$ of the medium (gain, absorption or refractive index) is modified. After that it relaxes then leading to a modulation of $\mathrm{X}$ along the round-trip period. This modulation produces the relative movement of pulses, resulting in pulse ordering along the cavity, and then leading to harmonic passive mode-locking. Both the gain modulation and the darkening absorber modulation lead to repulsion between pulses in the cavity. As a consequence, the identical pulses become uniformly distributed over the cavity length.

Figure 15 shows an example of ordering of pulses in the cavity by modulation of the net gain due to additional darkening absorber.

\section{IV - Conclusion}

In this paper, we have given an overview of our recent theoretical results concerning passively mode-locked fiber lasers. The most significant results are the demonstrations of both the cubic 
and the quintic CGL equations for passively mode-locked ring fiber lasers where the coefficients explicitly depend on physical parameters. This allowed us to successfully investigate the mode-locking properties for several cases of interest such as soliton fiber lasers, stretched-pulse fiber lasers or harmonic mode-locking. Our actual researches include an erbium-doped double-clad fiber laser and concern the interaction of a large number of pulses inside the laser cavity.

\section{Acknowledgments}

A.Komarov would like to thank the European Community which supports his research through a Marie Curie International Fellowship within the $6^{\text {th }}$ European Community Framework Programme. 


\section{References}

1. Bulushev, A.G., E.M. Dianov, and O.G. Okhotnikov. 1991. Self-starting modelocked laser with a nonlinear ring resonator. Opt. Lett. 16: 88.

2. Richardson, D.J., R.I. Laming, D.N. Payne, M.W. Phillips, and V.J. Matsas. 1991. $320 \mathrm{fs}$ soliton generation with passively mode-locked erbium fibre laser. Electron. Lett. 27: 730.

3. Richardson, D.J., R.I. Laming, D.N. Payne, V.J. Matsas, and M.W. Phillips. 1991. Pulse repetition rates in passive, self-starting, femtosecond soliton fibre laser. Eletron. Lett. 27: 1451.

4. Nakazawa, M., E. Yoshida, and Y. Kimura. 1991. Low threshold, 290 fs erbiumdoped fiber laser with a nonlinear amplifying loop mirror pumped by InGaAsP laser diodes. Appl. Phys. Lett. 59: 2073.

5. Grudinin, A.B., D.J. Richardson, and D.N. Payne. 1992. Energy quantization in figure eight fibre laser. Electron. Lett. 28: 67.

6. Guy, M.J., D.U. Noske, and J.R. Taylor. 1993. Generation of femtosecond soliton pulses by passively mode locking of an ytterbium-erbium figure-of-eight fibre laser. Opt. Lett. 18: 1447.

7. Guy, M.J., D.U. Noske, A. Boskovic, and J.R. Taylor. 1994. Femtosecond soliton generation in praseodymium fluoride fiber laser. Opt. Lett. 19: 828.

8. Avdokhin, A.V., S.V. Popov, and J.R. Taylor. 2003. Totally fiber integrated, figureof-eight, femtosecond source at 1065nm. Optics Express 11: 265.

9. Dahlström, L. 1972. Passive mode-locking and Q-switching of high power lasers by means of the optical Kerr effect. Opt. Com. 5: 157.

10. Chen, C.-J., P.K.A. Wai, and C. Menyuk, 1992. Soliton fiber ring laser. Opt. Lett. 17: 417.

11. Matsas, V.J., T.P. Newson, D.J. Richardson, and D.N. Payne. 1992. Self-starting passively mode-locked fibre ring soliton laser exploiting nonlinear polarisation rotation. Electron. Lett. 28: 1391.

12. Noske, D.U., N. Pandit, and J.R. Taylor. 1992. Subpicosecond soliton pulse formation from self-mode-locked erbium fibre laser using intensity dependent polarisation rotation. Electron. Lett. 28: 2185.

13. Tamura, K., H.A. Haus, and E.P. Ippen. 1992. Self-starting additive pulse modelocked erbium fibre ring laser. Eletron. Lett. 28: 2226. 
14. Tamura, K., E.P. Ippen, H.A. Haus, and L.E. Nelson. 1993. 77 fs pulse generation from a stretched-pulse mode-locked all-fiber ring laser. Opt. Lett. 18: 1080.

15. Tamura, K., C.R. Doerr, L.E. Nelson, H.A. Haus, and E.P. Ippen. 1994. Technique for obtaining high-energy ultrashort pulses from an additive-pulse mode-locked erbium-doped fiber ring laser. Opt. Lett. 19: 46.

16. Abedin, K.S., J.T. Gopinath, L.A. Jiang, M.E. Grein, H.A. Haus, and E.P. Ippen. 2002. Self-stabilized passive, harmonically mode-locked stretched-pulse erbium fiber ring laser. Opt. Lett. 27: 1758.

17. Tang, D.Y., W.S. Man, and H.Y. Tam. 1999. Stimulated soliton pulse formation and its mechanism in a passively mode-locked fibre soliton laser. Opt. Com. 165: 189.

18. Tang, D.Y., W.S. Man, H.Y. Tam, and P.D.Drummond. 2001. Observation of bound states of solitons in a passively mode-locked fiber laser. Phys. Rev. A 64: 033814.

19. Gong, Y., P. Shum, T. Hiang, Cheng, Q. Wen, and D. Tang. 2001. Bound soliton pulses in passively mode-locked fiber laser. Opt. Com. 200: 389.

20. Tang, D.Y., B. Zhao, D.Y. Shen, C. Lu, W.S. Man, and H.Y. Tam. 2002. Bound soliton fiber laser. Phys. Rev. A 66: 033806.

21. Grelu, P., F. Belhache, F. Gutty, and J.-M. Soto Crespo. 2002. Phase-locked soliton pairs in a stretched-pulse fiber laser. Opt. Lett. 27: 966.

22. Hideur, A., B. Ortaç, T. Chartier, M. Brunel, H. Leblond, and F.Sanchez. 2003. Ultra-short bound states generation with a passively mode-locked high-power Ybdoped double-clad fiber laser. Opt. Com. 225: 71.

23. Grelu, P., and J.-M.Soto Crespo. 2004. Multisoliton states and pulse fragmentation in a passively mode-locked fibre laser. J. Opt. B: Quantum Semiclass. Opt. 6: 271.

24. Ortaç, B., A. Hideur, T. Chartier, M. Brunel, P. Grelu, H. Leblond, and F. Sanchez. 2004. Generation of bound states of three ultrashort pulses with a passively modelocked high-power Yb-doped double-clad fiber laser. IEEE Photon. Tech. Lett. 16, 1274.

25. Seong, N.H., and D.Y.Kim. 2002. Experimental observation of stable bound solitons in a figure-eight fiber laser. Opt. Lett. 27: 1321.

26. Gong, Y.D., P. Shum, D.Y. Tang, C. Lu, Z.W. Qi, W.J. Lai, W.S. Man, and H.Y. Tam. 2003. Close spaced ultra-short bound solitons from DI-NOLM figure-8 fiber laser. Opt. Com. 220: 297.

27. Haus, H.A., J.G. Fujimoto, and E.P .Ippen. 1991. Structures for additive pulse mode locking. J. Opt. Soc. Am. B 8: 2068. 
28. Haus, H.A., J.G. Fujimoto, and E.P .Ippen. 1992. Analytic theory of additive pulse and kerr lens mode locking. IEEE J. Quant. Electron. 28: 2086.

29. Haus, H.A., E.P. Ippen, and K. Tamura. 1994. Additive-pulse modelocking in fiber lasers. IEEE J. Quant. Electron. 30: 200.

30. Ippen, E.P. 1994. Principles of passive mode locking. Appl. Phys. B. 58: 159.

31. Nelson, L.E., D.J. Jones, K. Tamura, H.A .Haus, and E.P. Ippen. 1997. Ultrashortpulse fiber ring lasers. Appl. Phys. B 65: 277.

32. Kim, A.D., J.N. Kutz, and D.J. Muraki. 2000. Pulse-train uniformity in optical fiber lasers passively mode-locked by nonlinear polarization rotation. IEEE J. Quant. Electron. 36: 465.

33. Spaulding, K.M., D.H. Yong, A.D. Kim, and J.N.Kutz. 2002. Nonlinear dynamics of mode-locking optical fiber ring lasers. J. Opt. Soc. Am. B 19: 1045.

34. Leblond, H., M. Salhi, A. Hideur, T. Chartier, M. Brunel, and F.Sanchez. 2002. Experimental and theoretical study of the passively mode-locked ytterbium-doped double-clad fiber laser. Phys. Rev. A 65: 063811.

35. Salhi, M., H. Leblond, and F. Sanchez. 2003. Theoretical study of the erbium-doped fiber laser passively mode-locked by nonlinear polarization rotation. Phys. Rev. A 67: 013802 .

36. Salhi, M., H. Leblond, and F. Sanchez. 2003. Theoretical study of the stretchedpulse erbium-doped fiber laser. Phys. Rev. A 68: 033815.

37. Komarov, A., H. Leblond, and F. Sanchez. 2005. Multistability and hysteresis phenomena in passively mode-locked fiber lasers. Phys. Rev. A 71: 053809.

38. Komarov, A., H. Leblond, and F. Sanchez. 2005. Quintic complex Ginzburg-Landau model for ring fiber lasers. Phys. Rev. E 72: 025604(R).

39. Akhmediev, N.N., A. Ankiewicz, and J.-M. Soto-Crespo. 1997. Miltisoliton solutions of the complex Ginzbug-Landau equation. Phys. Rev. Lett. 79: 4047.

40. Akhmediev, N.N., A. Ankiewicz, and J.-M. Soto-Crespo. 1998. Stable soliton pairs in optical transmission lines and fiber lasers. J. Opt. Soc. Am. B 15: 515.

41. Komarov, A., H. Leblond, and F. Sanchez. 2006. Passive harmonic mode-locking in a fiber laser with nonlinear polarization rotation. Opt. Com. 267: 162.

42. Hideur, A., T. Chartier, M. Brunel, M. Salhi, C. Özkul, and F. Sanchez. 2001. Modelock, $Q$-switch and CW operation of an ytterbium-doped double-clad fiber ring laser. Opt. Com. 198: 141.

43. Agrawal, G.P. 1995. Nonlinear Fiber Optics, New York: Academic Press. 
44. Taniuti T., and C.C. Wei. 1968. Reductive perturbation method in nonlinear wave propagation I. J. Phys. Soc. Jap. 24: 941.

45. Komarov, K.P. 1986. Theory of stationary ultrashort pulses in solid-state lasers with passive mode-locking. Opt. and Spec. 60: 213.

46. Komarov, K.P., A.S. Kuch'yanov, and V.D. Ugozhaev. 1986. Generation of stationary ultrashort pulses by passively mode-locking solid-state lasers. Opt. Com. 57: 279.

47. Soto-Crespo, J.-M., N.N. Akhmediev, V.V. Afanasjev, and S. Wabnitz. 1997. Pulse solutions of the cubic-quintic complex Ginzburg-Landau equation in the case of normal dispersion. Phys. Rev. E 55: 4783.

48. Ortaç, B., A. Hideur, M. Brunel, T. Chartier, M. Salhi, H. Leblond, and F. Sanchez. 2003. Characterization of an ytterbium-doped double-clad fiber laser passively mode-locked by nonlinear polarization rotation. Appl. Phys. B 77: 589.

49. Akhmediev N.N., and A. Ankiewicz. 1997. Solitons, Nonlinear Pulses and Beams, London: Chapman and Hall.

50. Akhmediev N.N., and V.V. Afanasjev. 1995. Novel arbitrary-amplitude soliton solutions of the cubic-quintic complex Ginzburg-Landau equation. Phys. Rev. Lett. 75: 2320.

51. Haus, H.A., K. Tamura, L.E. Nelson, and E.P. Ippen. 1995. Stretched-pulse additive pulse mode-locking in fiber ring lasers: theory and experiment. IEEE J. Quant. Electron. 31: 591.

52. Salhi, M., H. Leblond, F. Sanchez, M. Brunel, and A. Hideur. 2004. Stability calculations for the ytterbium-doped fibre laser passively mode-locked through nonlinear polarization rotation. J. Opt. A: Pure and Appl. Opt. 6: 774.

53. Grelu, P., F. Belhache, F. Gutty, and J.M. Soto-Crespo. 2003. Relative phase locking of pulses in a passively mode-locked fiber laser. J. Opt. Soc. Am. B 20: 863.

54. Zhao, B., D.Y. Tang, L.M. Zhao, P. Shum, and H.Y .Tam. 2004. Pulse-train nonuniformity in a fiber ring laser mode-locked by using the nonlinear polarization rotation technique. Phys. Rev. A 69: 043808.

55. Tang, D.Y., L.M. Zhao, and B. Zhao. 2005. Soliton collapse and bunched noise-like pulse generation in a passively mode-locked fiber ring laser. Opt. Express 13: 2289.

56. Komarov, A., H. Leblond, and F. Sanchez. 2005. Theoretical analysis of the operating regime of a passively mode-locked fiber laser through nonlinear polarization rotation. Phys. Rev. A 72: 063811. 
57. Haboucha, A., M. Salhi, A. Komarov, H. Leblond, and F. Sanchez. 2006. Influence of the nonlinear polarization rotation on a nonlinear fiber loop. J. Non. Opt. Phys. Mat. 15: 157.

58. Digonnet, M.J.F., Ed.. 2001. Rare-earth-doped fiber lasers and amplifiers, New York: Marcel Dekker

59. Afanasjev, V.V., N. Akhmediev, and J.M. Soto-Crespo. 1996. Three forms of localized solutions of the quintic complex Ginzburg-Landau equation. Phys. Rev. E 53: 1931.

60. Leblond, H., A. Komarov, M. Salhi, A. Haboucha, and F. Sanchez. 2006. Cis bound states of three localized pulses of the cubic-quintic CGL equation. J. Opt. A: Pure Appl. Opt. 8: 319.

61. Soto-Crespo, J.M., Ph. Grelu, N. Akhmediev, and N. Devine. 2007. Soliton complexes in dissipative systems: vibrating, shaking and mixed soliton pairs. Phys. Rev. E 75: 016613.

62. Yu, C.X., H.A. Haus, E.P. Ippen, W.S. Wong, and A. Sysiolatin. 2000. Gigahertzrepetition-rate mode-locked fiber laser for continuum generation. Opt. Lett. 25: 1418.

63. Carruthers, T.F., I.N. Duling III, M. Horowitz, and C.R. Menyuk. 2000. Dispersion management in a harmonically mode-locked fiber soliton laser. Opt. Lett. 25: 153.

64. Grudinin A.B., and S.Gray. 1997. Passive harmonic mode locking in soliton fiber lasers. J. Opt. Soc. Am. B 14: 144.

65. Ortaç, B., A. Hideur, and M. Brunel. 2004. Passive harmonic mode locking with a high-power ytterbium-doped double-clad fiber laser. Opt. Lett. 29: 1995.

66. Brunel, M., C. Özkul, and F. Sanchez. 1999. Dynamics of a laser with a reverse saturable absorber. Appl. Phys. B 68: 39. 


\section{Figure captions}

Figure 1: Schematic representation of the laser. $\mathrm{P}$ is the polarizer, $\theta_{ \pm}$are the orientation angles of the eigenaxis of the fiber with respect to the passing axis of the polarizer and $f_{n}$ is the electric field amplitude after the polarizer after the $n^{\text {th }}$ round-trip.

Figure 2: Schematic representation of the effect of the nonlinear gain $\mathcal{D}_{i}$, of the excess of linear gain $g_{1}$, and of both the dispersion $\beta_{2}$ and the effective self-phase modulation $\mathcal{D}_{\mathrm{r}}$ on a localized pulse.

Figure 3: Operating regime of the laser as a function of the angles $\theta_{+}$and $\theta_{-}$between the polarizer and the eigenaxis of the fiber, according to the theory for the normal dispersion regime. ML stands for mode-locked and CW for continuous wave regime.

Figure 4: Stability diagram of the constant nonzero and localized solutions in the plane $(\mathrm{R}, \mathrm{D})$.

Figure 5: Operating regime of the laser as a function of the angles $\theta_{+}$and $\theta_{-}$between the polarizer and the eigenaxis of the fiber, according to the theory for the anomalous dispersion regime. The white region corresponds to stable mode-locking. In the grey region, the constant nonzero solution is stable while the stability of the mode-locked solution is not known. In the hatched domain, both solutions are unstable.

Figure 6: Evolution of the pulse energy in the plane $\left(\theta_{+}, \theta_{-}\right)$for the anomalous dispersion case. In the hatched region, the energy is above $10 \mathrm{pJ}$, in the grey region the energy is between 5 and $10 \mathrm{pJ}$ and in the black region the energy is below $5 \mathrm{pJ}$.

Figure 7: Evolution of the pulse duration in the plane $\left(\theta_{+}, \theta_{-}\right)$for the anomalous dispersion case. In the hatched region, the duration is above $1 \mathrm{ps,}$ in the grey region it is between 0.5 and $1 \mathrm{ps}$ and in the black region below $0.5 \mathrm{ps}$. 
Figure 8: Schematic representation of a fiber ring laser passively mode-locked through nonlinear polarization rotation. $\lambda / 2$ is a halfwave plate. $\lambda / 4$ is a quarterwave plate. The $\alpha_{i}$ 's are the angles between the one eigenaxis of the waveplate and the passing axis of the polarizer.

Figure 9: Multiple pulse operation. The parameters used are $\alpha_{3}=-\alpha_{1}=0.2, \alpha=-0.7$, $\mathrm{a}=1.2$ and $\mathrm{d}_{\mathrm{r}}=0.2$.

Figure 10: Hysteresis dependence of the lasing regime and of the number of pulses in steadystate operation versus the pumping parameter $\mathrm{a}$ in the normal dispersion regime $\mathrm{D}_{\mathrm{i}}=-1$. The parameters used are $\alpha=0.2, \alpha_{1}=-0.9, \alpha_{3}=0.2$ and $d_{r}=0.2$.

Figure 11: Hysteresis dependence of the number of pulses in the cavity $\mathrm{N}$, versus the orientation angles of the phase plates. The parameters used are $\mathrm{a}=2, \alpha_{1}=2, \alpha_{3}=0.2$ and $\mathrm{d}_{\mathrm{r}}=0.2$.

Figure 12: Evolution of the integrated intensity $J$ as a function of the round-trip number $\zeta$. (a) Undamped spike regime, (b) $\mathrm{CW}$ operation. The parameters used are $\mathrm{a}=2.5, \alpha=0.1$, $\alpha_{1}=-1.9, \alpha_{3}=0.2$ and $D_{r}=0.2$.

Figure 13: Hysteresis dependence of the operating regime of the laser as a function of the pumping parameter a. The parameters used are the same as in figure 12. QS: Q-switch operation, PML: polarization mode-locking regime and $\mathrm{CW}$ : continuous regime.

Figure 14: Propagation of a stable bound state of three pulses. The parameters are given in the text.

Figure 15: Effect of passive net gain modulation due to a darkening absorber. The parameters used are $\alpha=-1, \alpha_{1}=0.2, \alpha_{3}=0.3, D_{i}=-1, D_{r}=0.2, a=1.8, \tilde{a}=0.2, \tau_{r}=100$. 


\section{Biographies of the authors}

François Sanchez was born in Paris in 1962 and he obtained his PhD in 1987 from University of Paris Sud (Thales, Laboratoire Central de Recherche). The subject of the thesis was the investigation of wave mixing in liquid crystals and semiconductors in the middle infrared. In 1988, he obtained an assistant Professor position at ENSSAT where he was involved in several projects on fiber lasers and microchip lasers. He became Professor in 1997 at the University of Rouen where he developed the activities on double-clad fiber lasers. In 1999 he obtained the prize Arnulf Françon from the Société Française d'Optique (French Optical Society) for his book Optique Non-Linéaire. In 2000 he joined the laboratoire POMA at the University of Angers where his researches include nonlinear optics, fiber lasers, short pulse generation and soliton's interaction.

Hervé Leblond was born in Cahors, France, in 1964. He entered the Ecole Normale Supérieure, Paris, France, in 1983, received the Ph.D. degree from the University of Montpellier, Montpellier, France, in 1994 and a second Ph.D. degree from the University of Angers, Angers, France, in 2001. He has worked since 1996 at the Laboratory of the Optical Properties of Materials and Applications (POMA), University of Angers. He has studied several developments in the reductive perturbation method and applications to electromagnetic solitons in ferromagnetic materials and nonlinear optics. He is now also interested in fiber lasers and non-conservative solitons.

Mohamed SALHI was born in 1974 in Morocco. After a master's degree at the University of Mohamed 1 in Oujda he continued his studies at the University of Rouen in France and he obtained a $\mathrm{PhD}$ in Angers in 2004. The $\mathrm{PhD}$ was dedicated to the modelling of passively mode-locked fiber lasers through nonlinear polarization rotation. After that, he joined Multitel in Belgium as a research engineer where he participated to the development of compact shortpulse fiber lasers. He is actually a lecturer at the University of Angers. His subjects of interest concern passively mode-locked fiber lasers.

Andrey K. Komarov was born in Novosibirsk, Russia in 1977. He received the Bachelor Degree (1998) and the Magister Degree (2000), both in physics, from Novosibirsk State University. He received the Ph.D. (2003) in speciality "Optics" from the Institute of Automation and Electrometry, Russian Academy of Sciences. Since 2000 he is assistant 
professor in the Novosibirsk State University and permanent researcher at Institute of Automation and Electrometry (Laboratory of Nonlinear Physics). He has obtained a postdoctoral position at Laboratoire POMA, Université d'Angers, France in 2004-2005 and 2007-2009. The main field of interests is nonlinear dynamics of light in lasers and various nonlinear systems. He is a specialist on numerical simulation of nonlinear optical systems. With colleagues, he developed the theory of laser passive mode-locking which describes the quantization of radiation into identical dissipative solitons, multistability and multihysteresis phenomena, threshold self-start of passive mode-locking and other effects. He is author of more than 50 publications.

Adil Haboucha was born in Oujda, Morocco, in 1974. He received the Engineer degree in Optronics from the Ecole Nationale Supérieure des Sciences Appliquées et de Technologie (ENSSAT, Lannion), France. He obtained the DEA degree from the University of Angers. He is actually finalizing his $\mathrm{PhD}$ work which is dedicated to the investigation of the interaction of a very large number of solitons in a fiber laser cavity. 


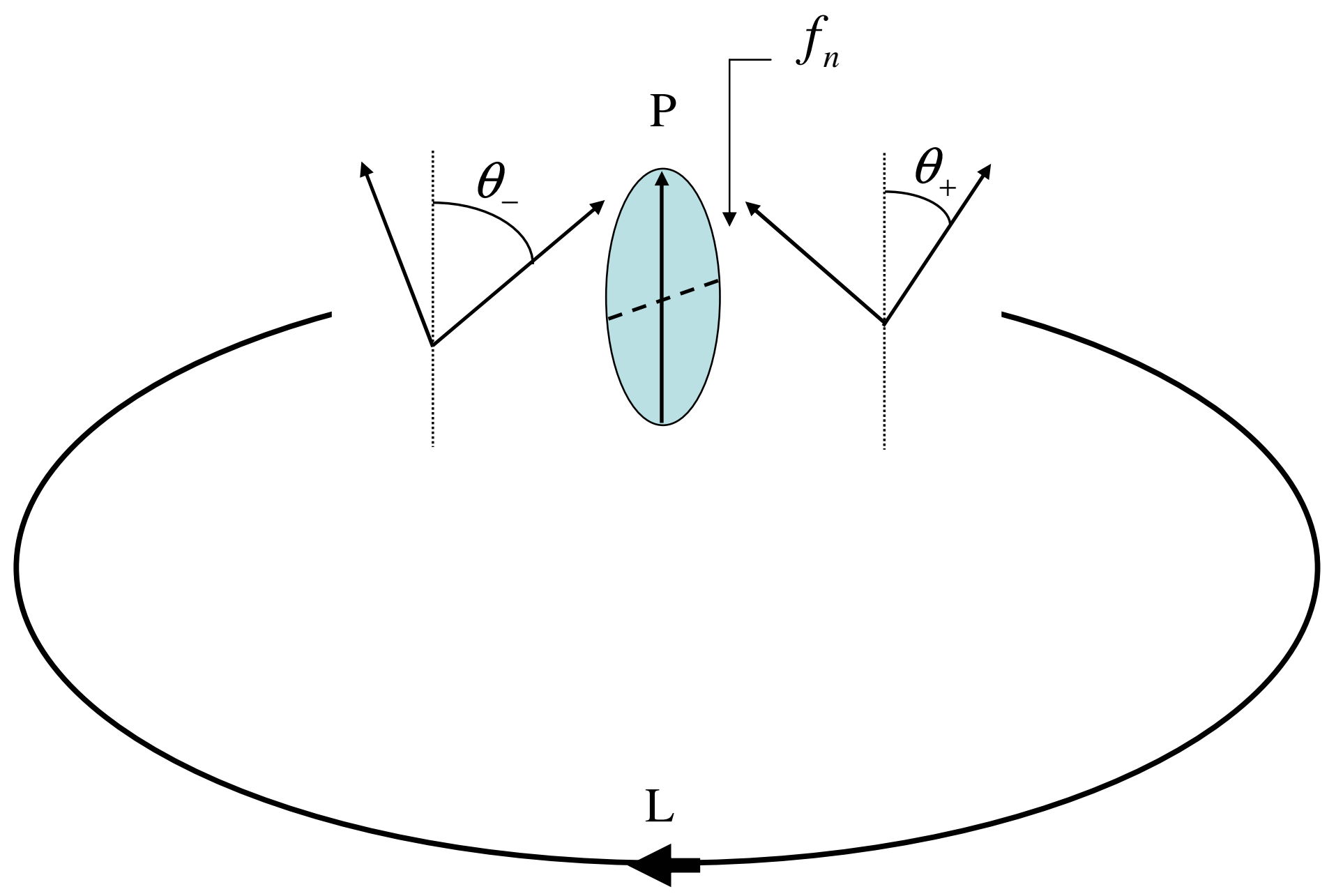

Figure 1 
(a)

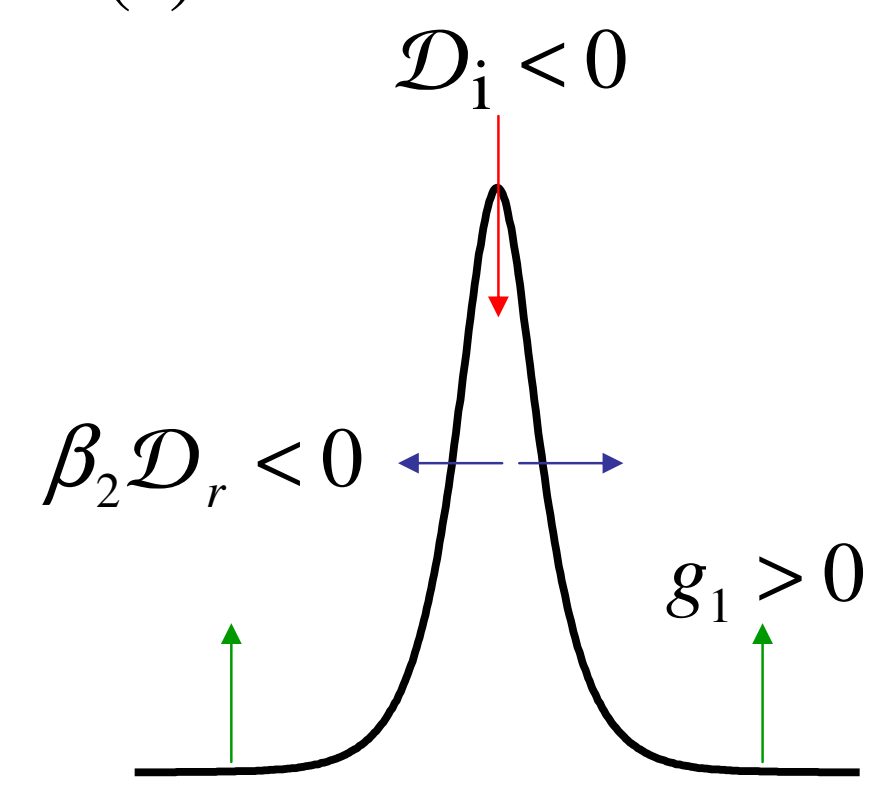

(b)

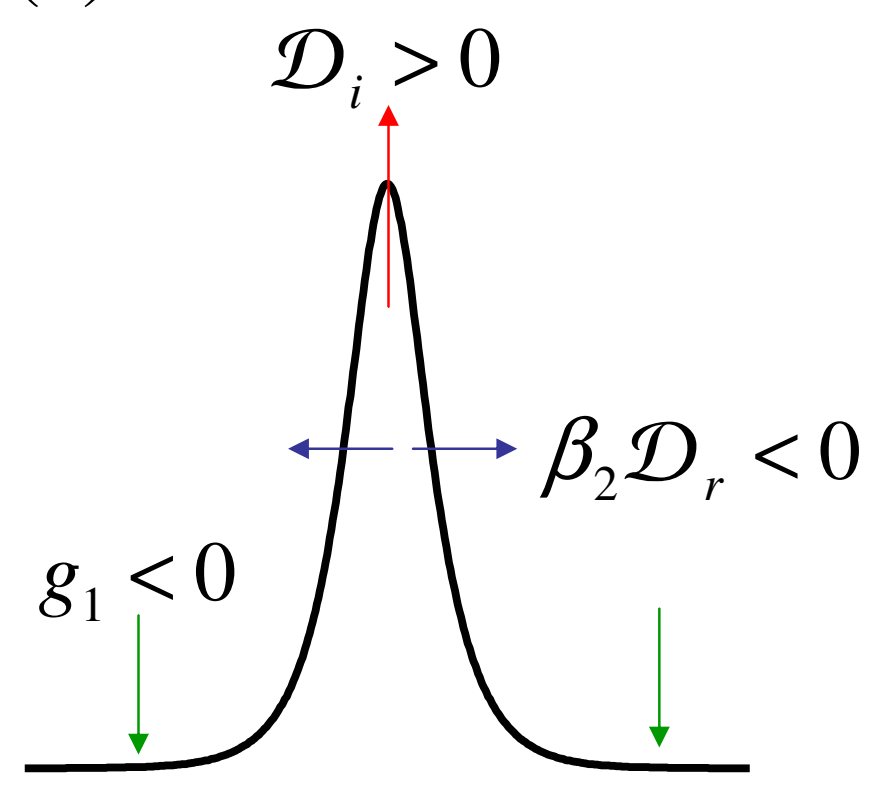

Figure 2 


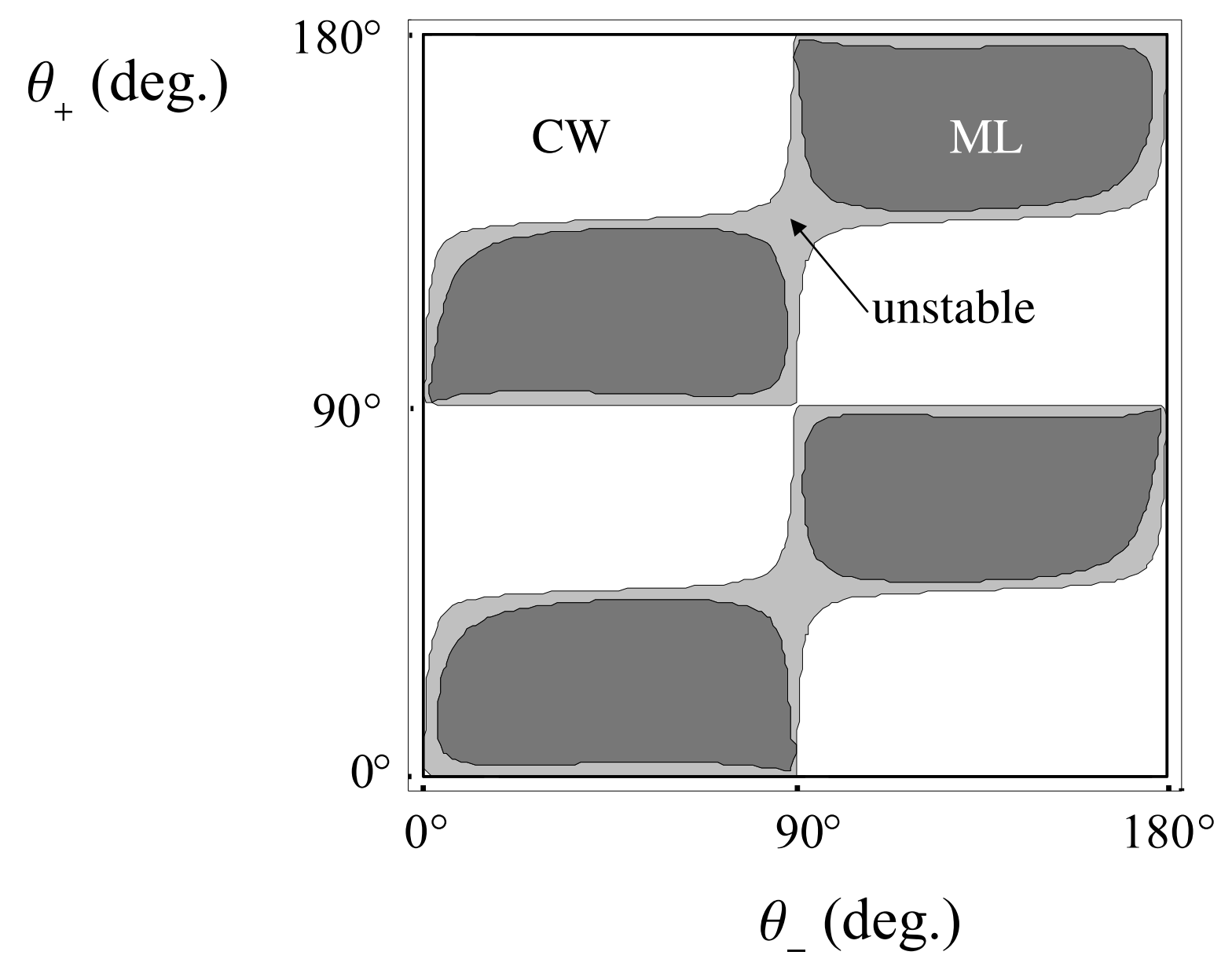

Figure 3 


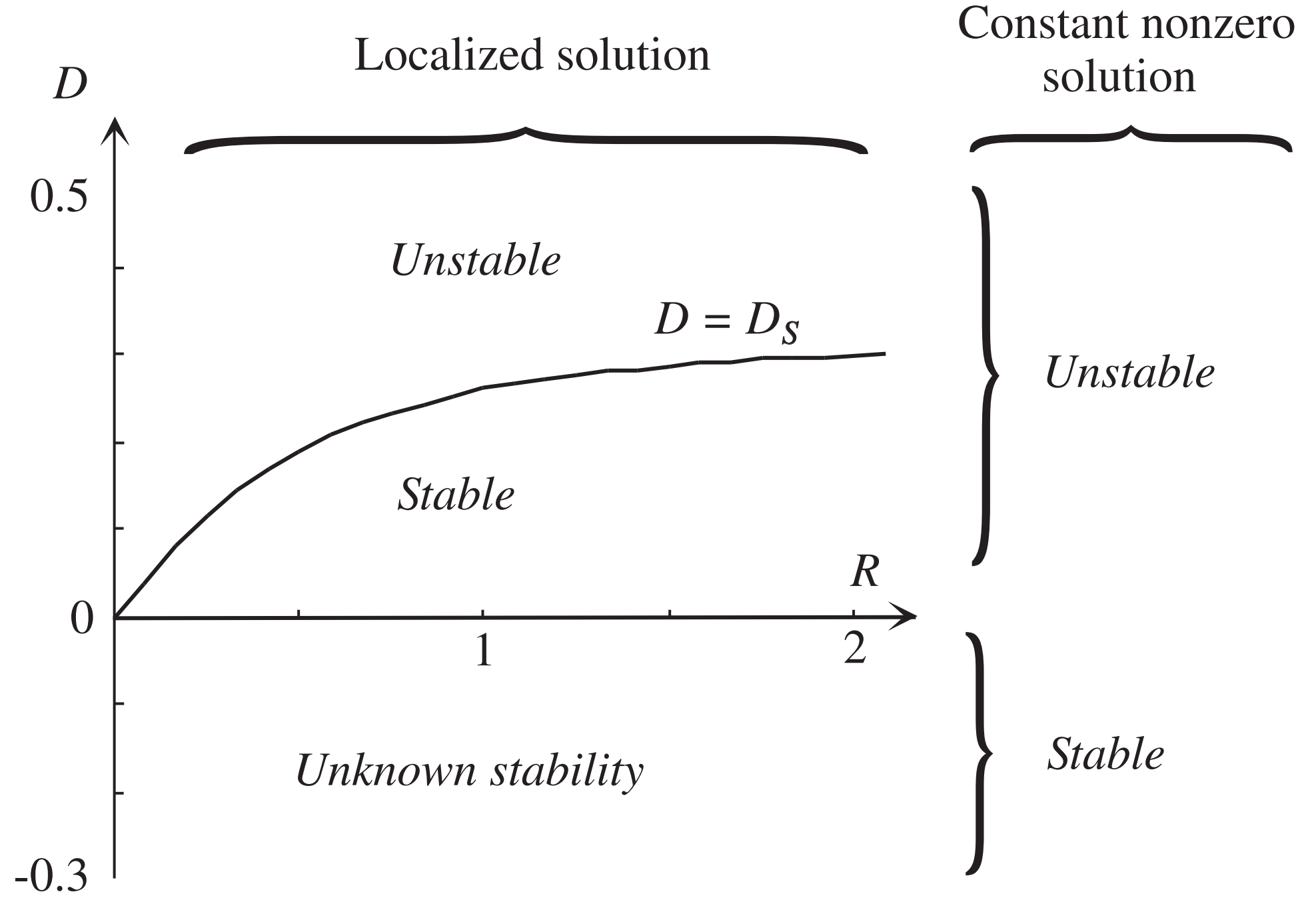

Figure 4 


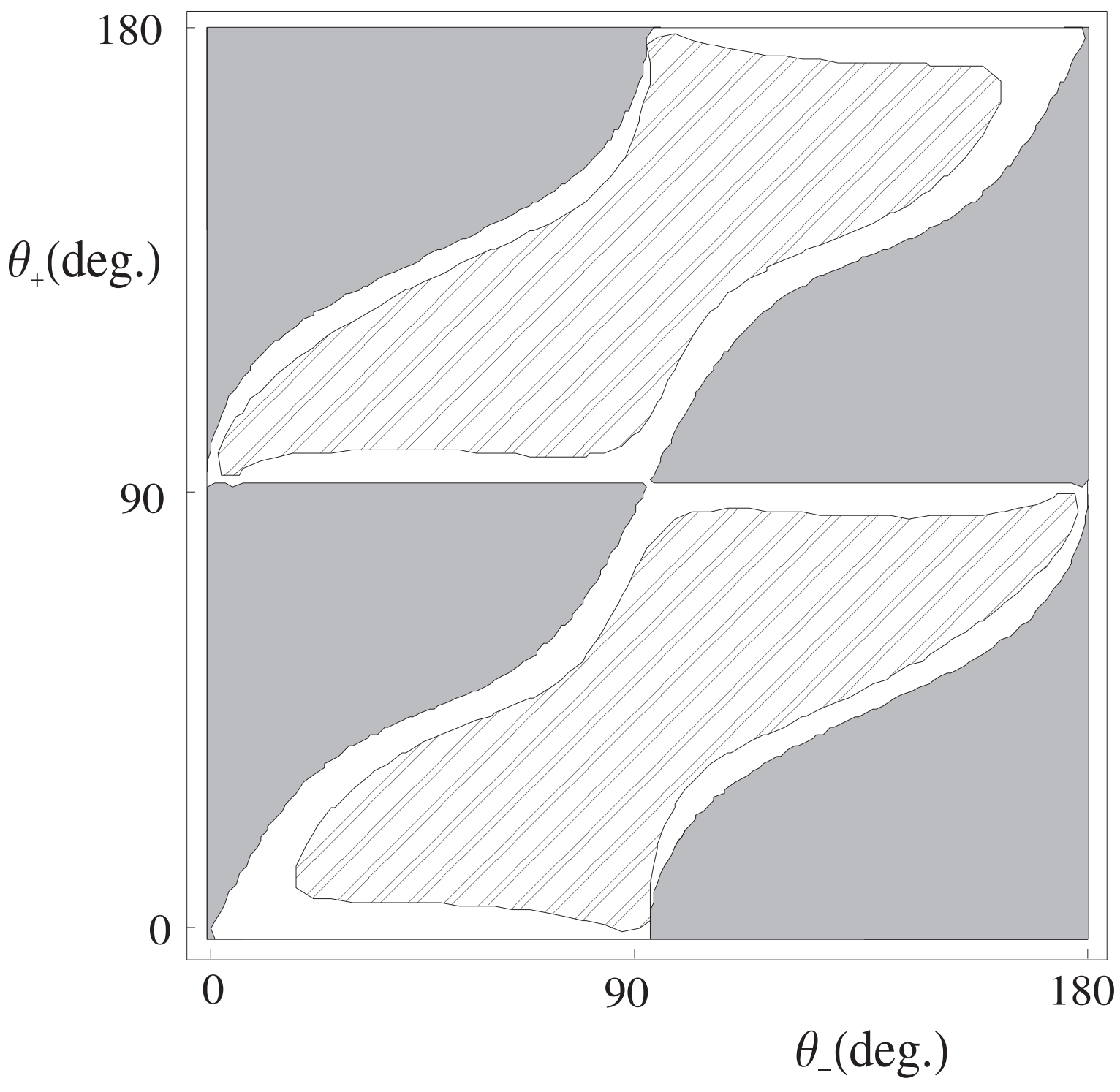

Figure 5 


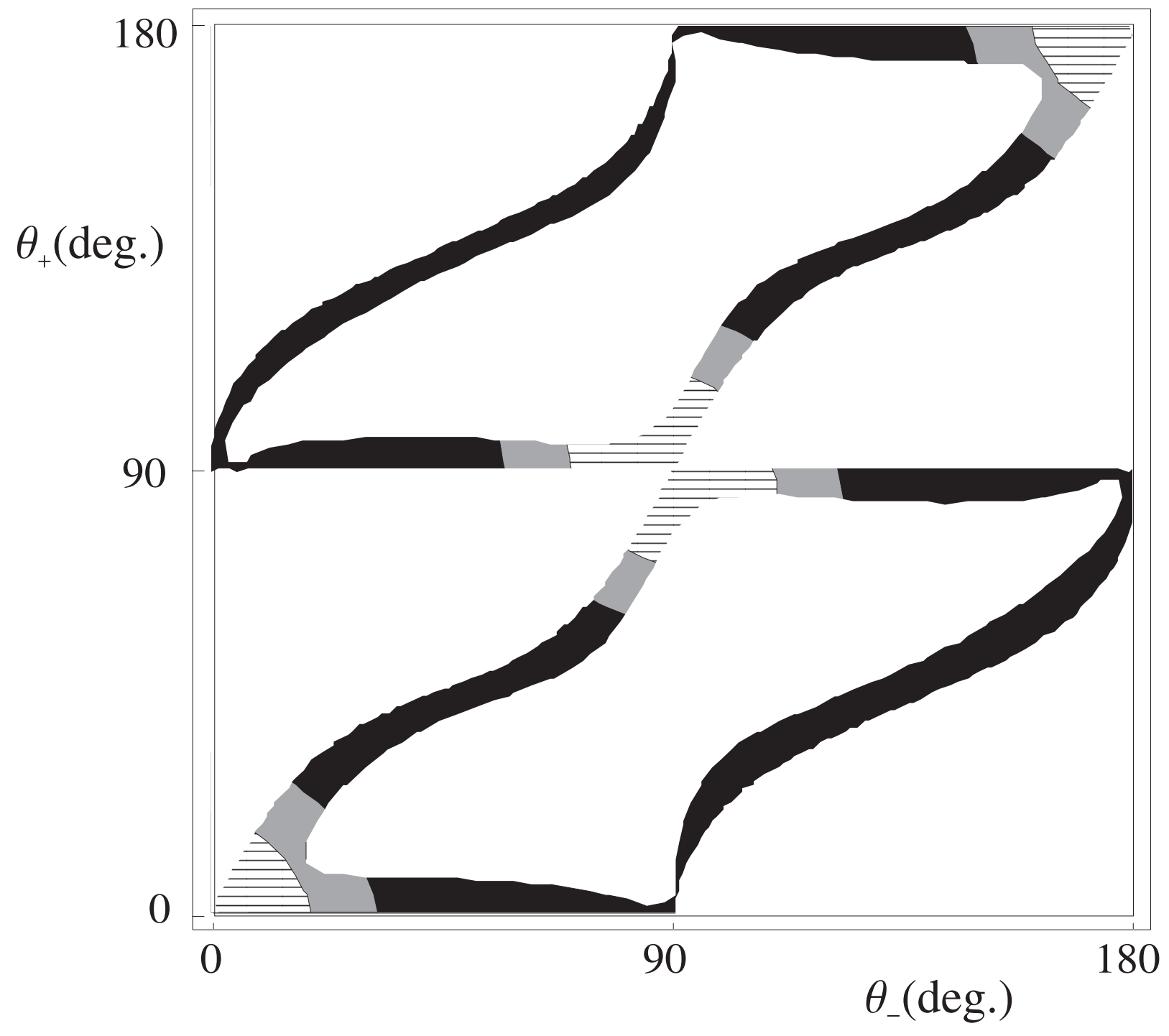

Figure 6 


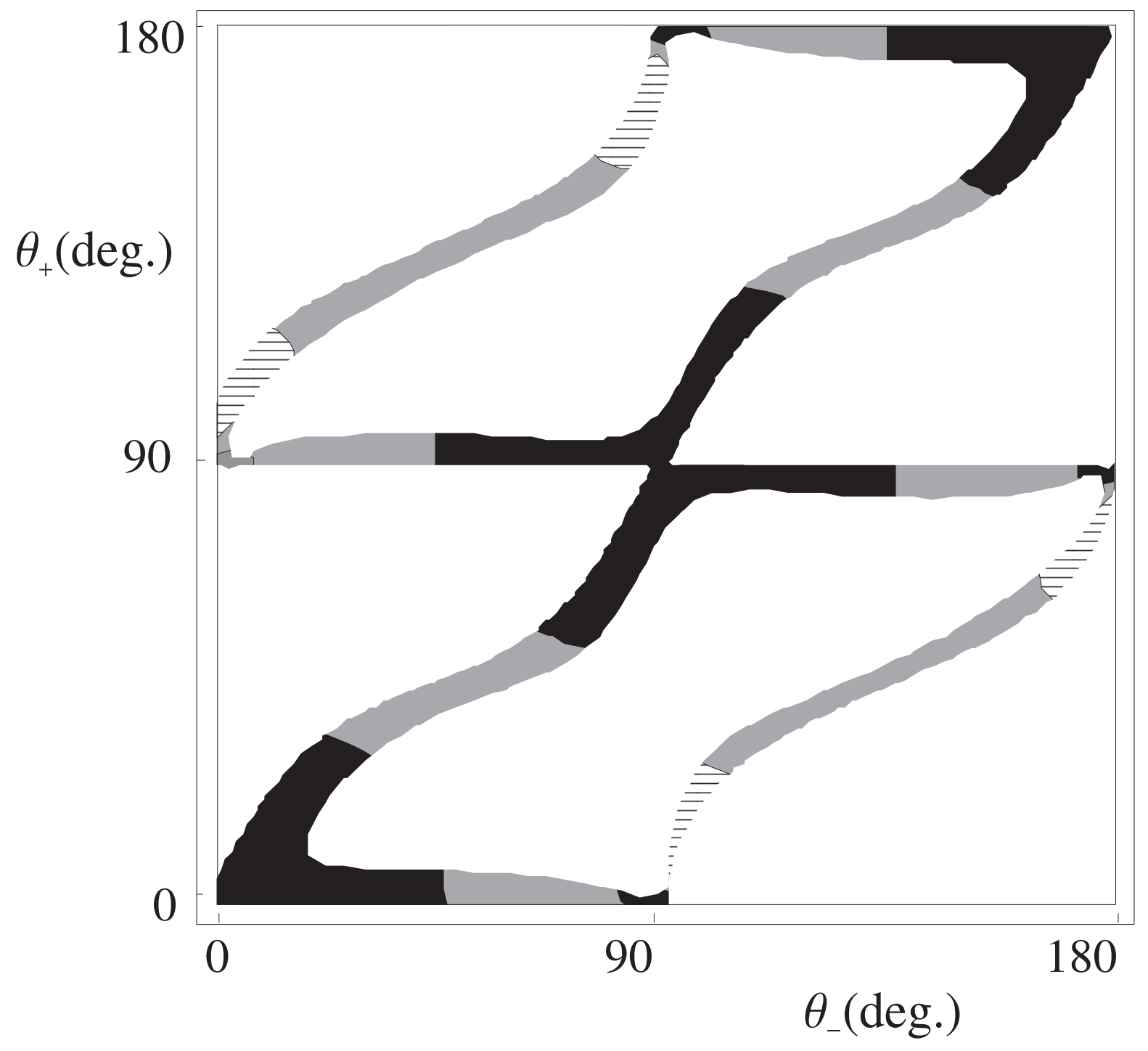

Figure 7 


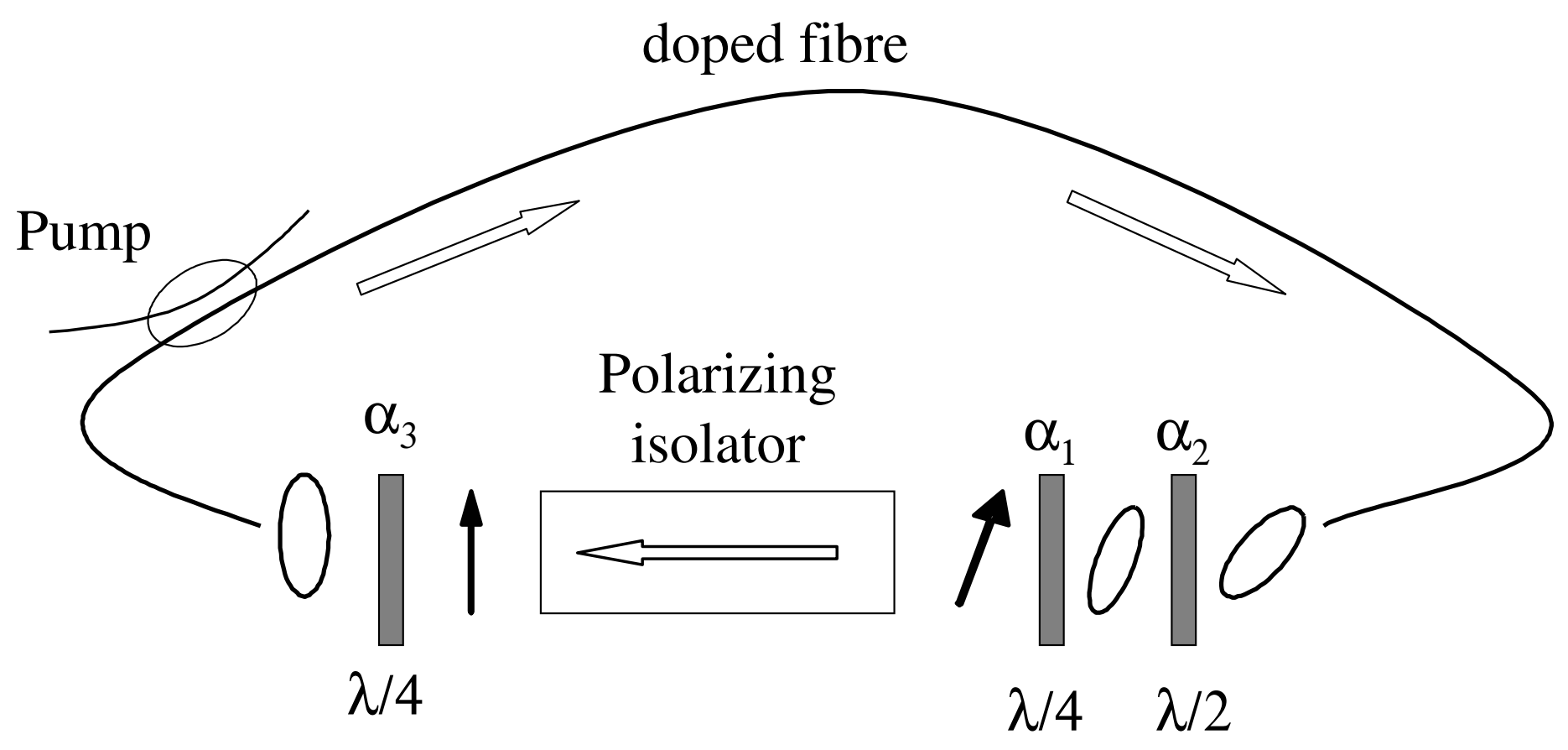

Figure 8 


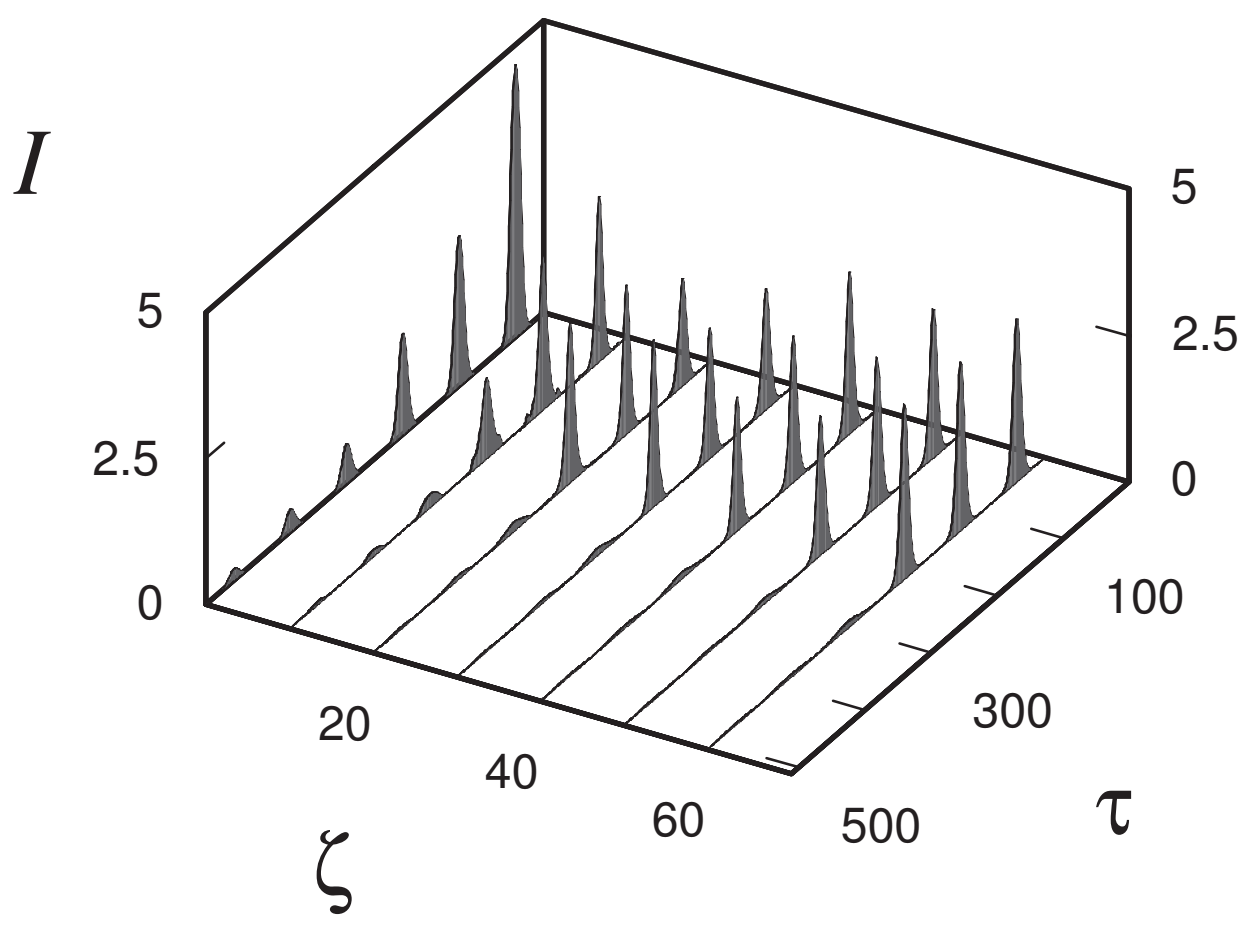

Figure 9 


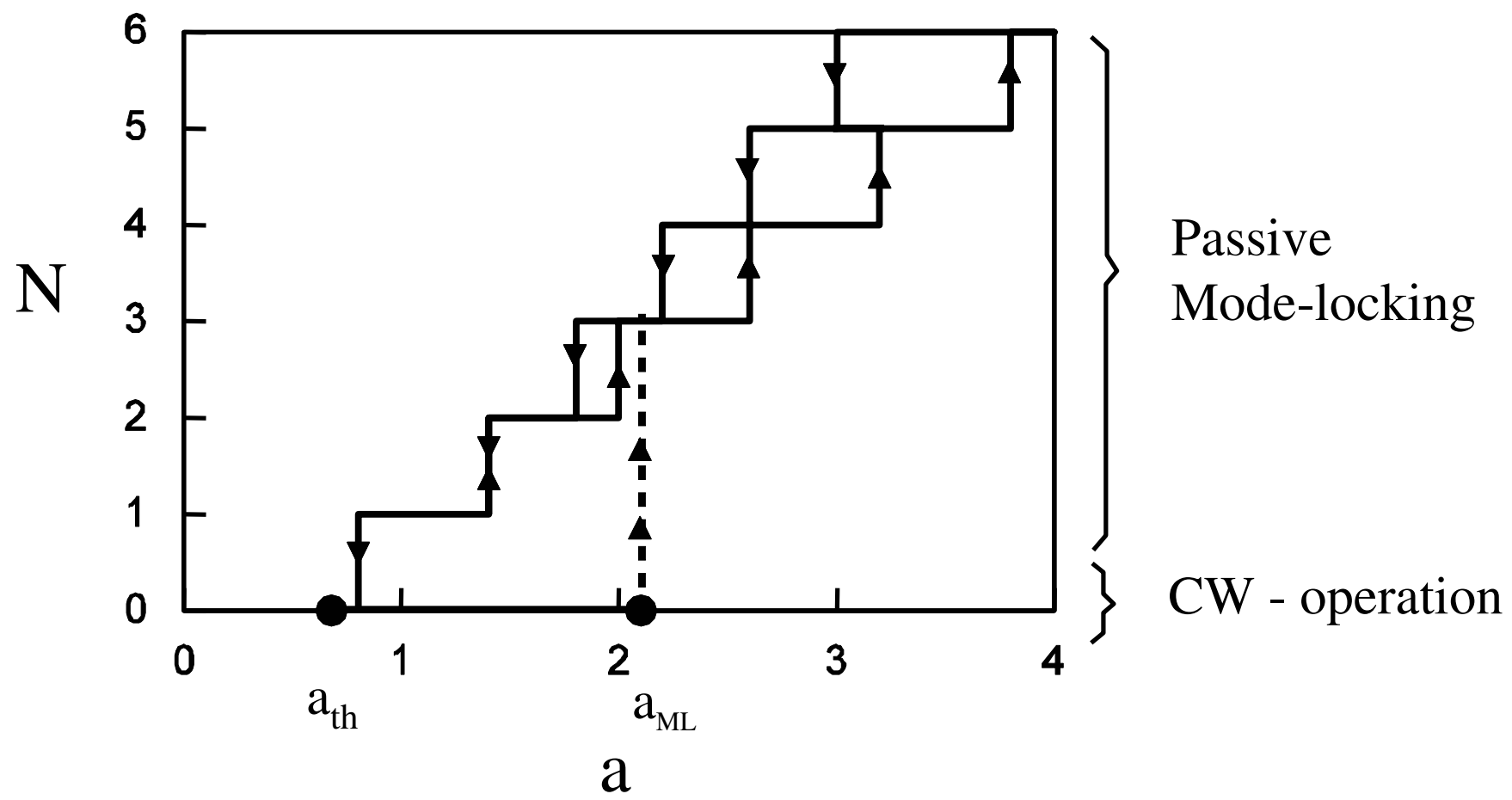

Figure 10 


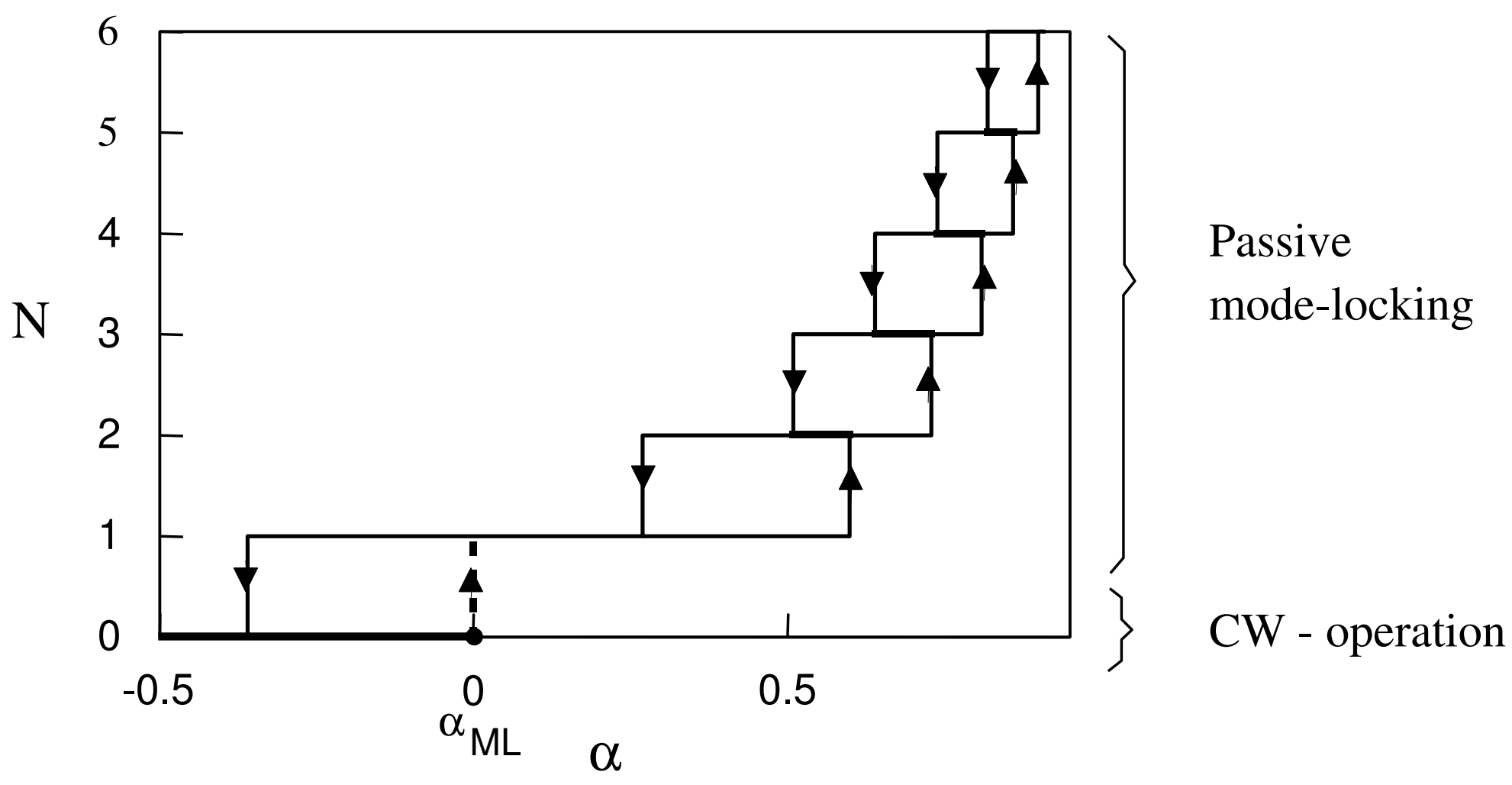

Figure 11 
(a)

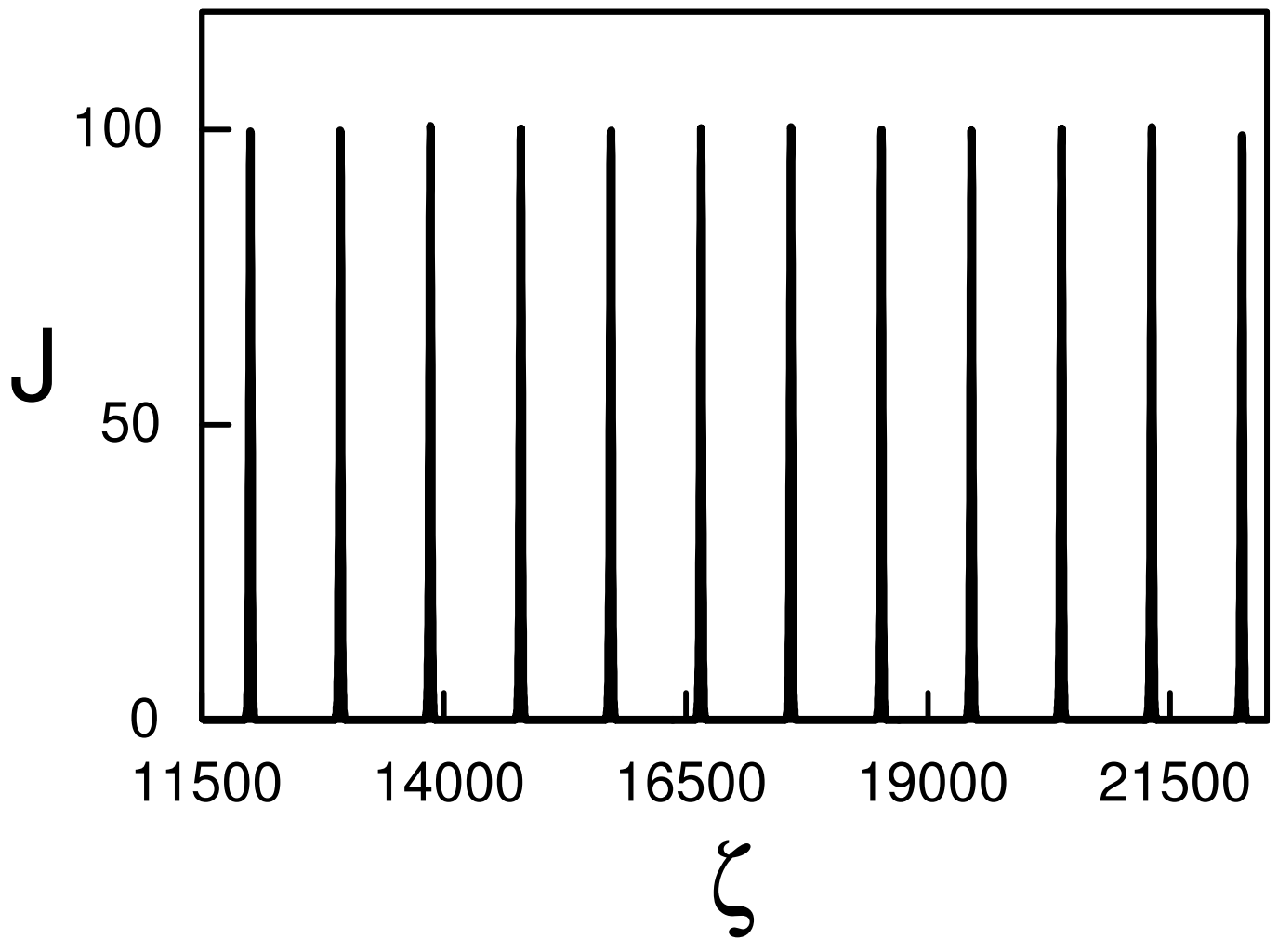

Figure 12(a) 
(b)

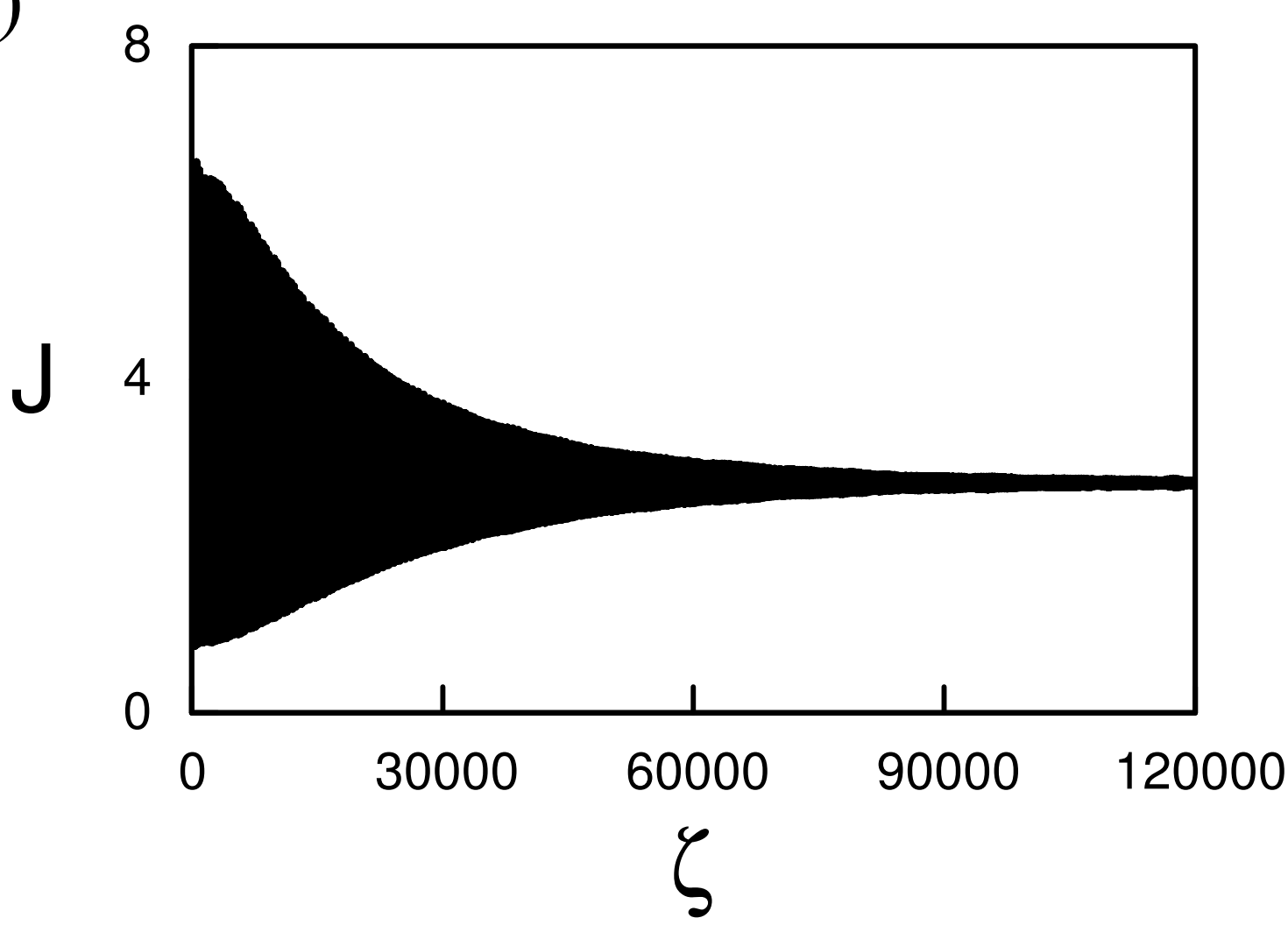

Figure 12(b) 


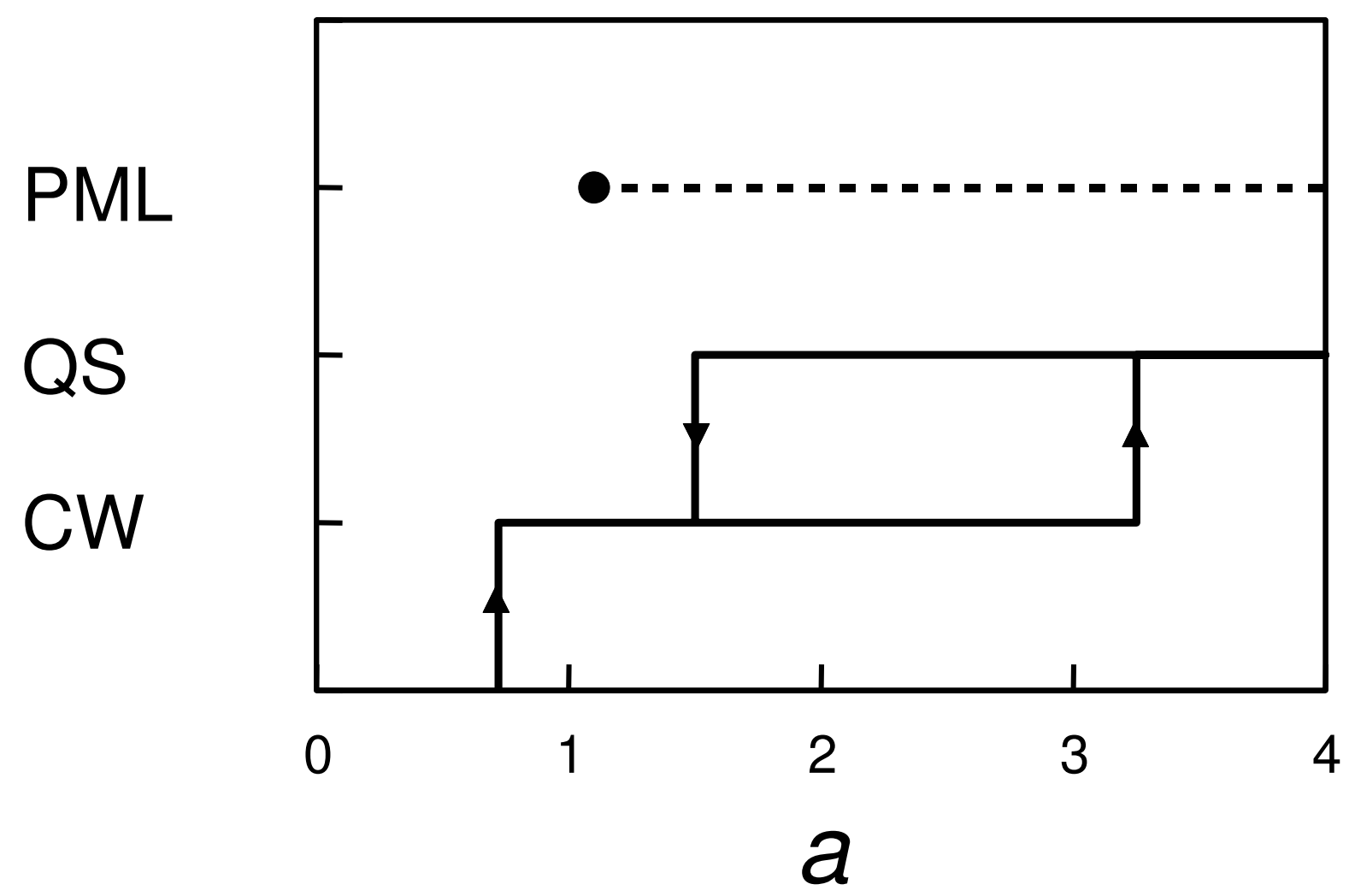

Figure 13 


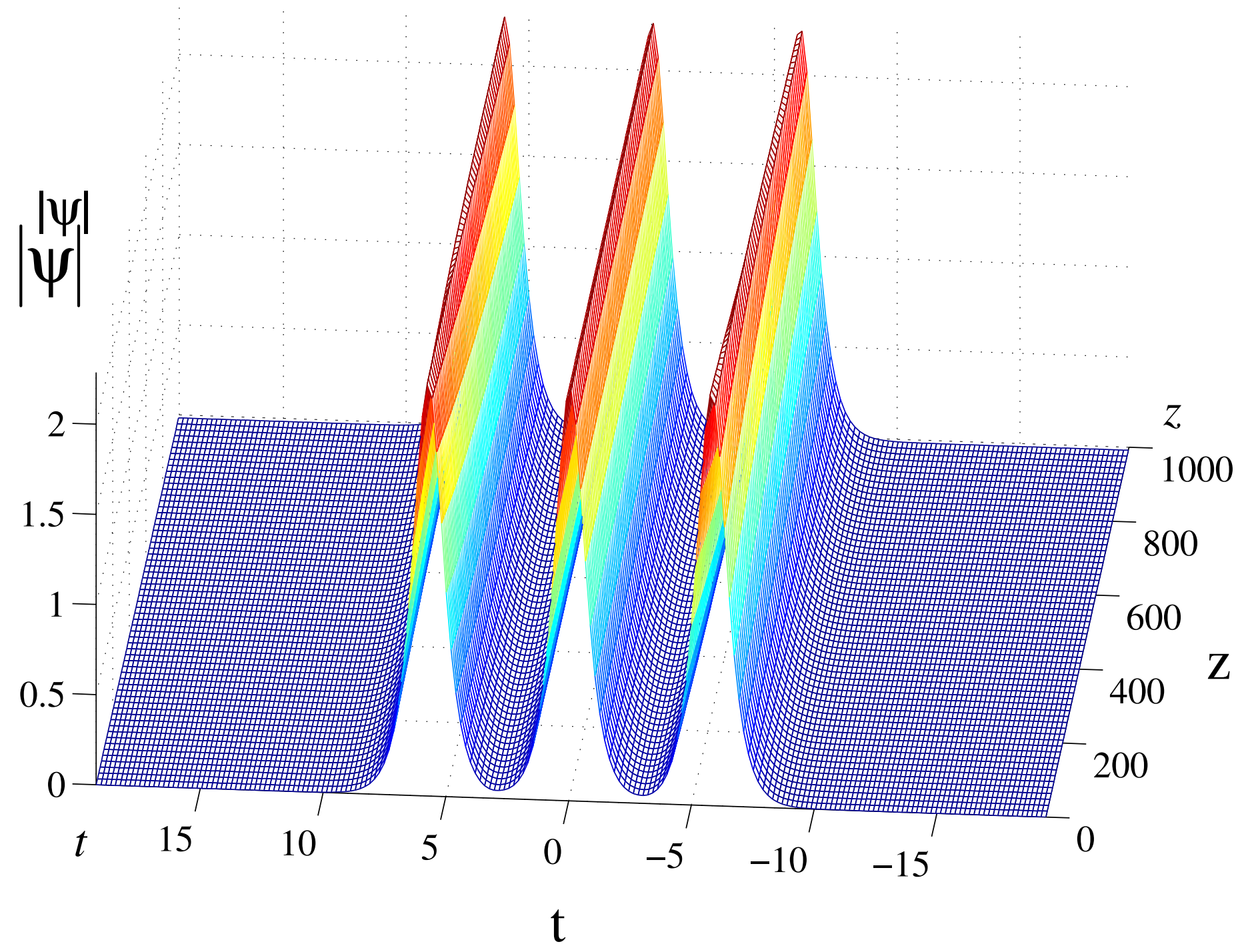

Figure 14 


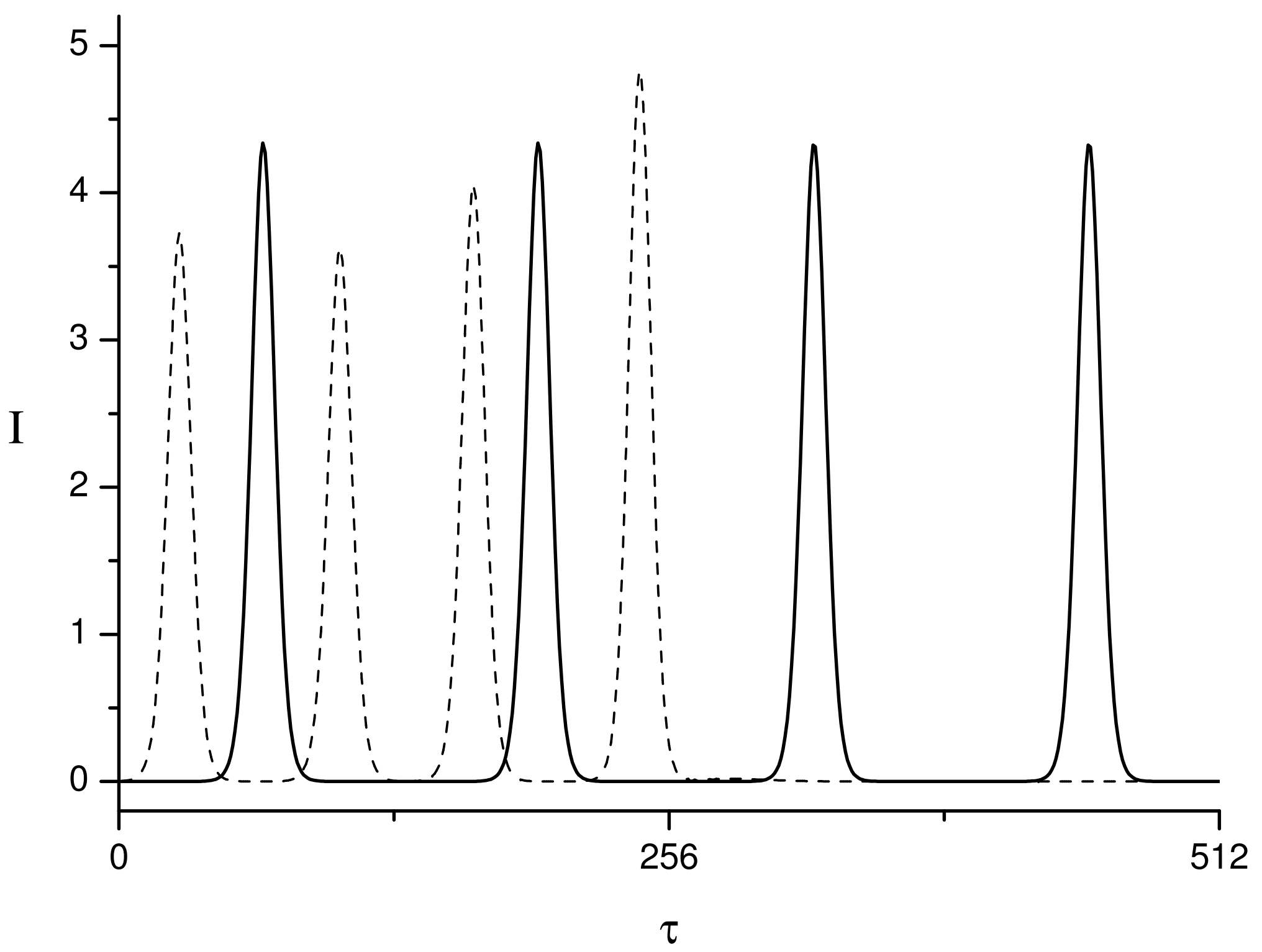

Figure 15 